\title{
A NOVEL EXTENSION OF TOPSIS WITH INTERVAL TYPE-2 TRAPEZOIDAL NEUTROSOPHIC NUMBERS USING $(\alpha, \beta, \gamma)$-CUTS
}

\author{
Muhammad Touqeer $^{1}$, Rimsha Umer ${ }^{1}$, Ali Ahmadian ${ }^{2,3, *} \odot$ \\ AND SoHeIl SAlahShour ${ }^{4}$
}

\begin{abstract}
Multi-criteria decision-making (MCDM) is concerned with structuring and solving decision problems involving multiple criteria for decision-makers in vague and inadequate environment. The "Technique for Order Preference by Similarity to Ideal Solution" (TOPSIS) is one of the mainly used tactic to deal with MCDM setbacks. In this article, we put forward an extension of TOPSIS with interval type-2 trapezoidal neutrosophic numbers (IT2TrNNs) using the concept of $(\alpha, \beta, \gamma)$-cut. First, we present a novel approach to compute the distance between two IT2TrNNs using ordered weighted averaging (OWA) operator and $(\alpha, \beta, \gamma)$-cut. Subsequently, we broaden the TOPSIS method in the context of IT2TrNNs and implemented it on a MCDM problem. Lastly, a constructive demonstration and several contrasts with the other prevailing techniques are employed to articulate the practicability of the proposed technique. The presented strategy yields a flexible solution for MCDM problems by considering the attitudes and perspectives of the decision-makers.
\end{abstract}

Mathematics Subject Classification. 06Dxx, 06D72.

Received March 15, 2021. Accepted August 9, 2021.

\section{INTRODUCTION}

"Multi-criteria decision-making" (MCDM) is a branch of operational research yielding results to rank and assess the best alternatives from set of alternatives under multiple criteria regarding decision-maker's choices and preferences [8]. In the conventional techniques for MCDM, the alternative ratings and criterion weights were articulated by using crisp values. Nevertheless, decision-makers frequently use natural language to eloquent their outlook and subjective observations in the existent world and it's complex to state the linguistic terms exactly and accurately by using crisp values. Consequently, the "fuzzy set" (FS) theory is introduced by Zadeh [57] to cope up with the problems involving subjective uncertainties. Afterwards, "type-2 fuzzy sets" (T2FSs) [26-28], an extension of "type-1 fuzzy sets" (T1FSs) were commenced since they can engage with more uncertainties

Keywords. Multi-criteria decision making (MCDM), interval type-2 trapezoidal neutrosophic number (IT2TrNN), ordered weighted averaging (OWA) operator.

1 Department of Basic Sciences, University of Engineering and Technology, Taxila, Pakistan.

2 Institute of IR 4.0, The National University of Malaysia, 43600 Bangi, Selangor, Malaysia.

3 Department of Mathematics, Near East University, TRNC, Mersin 10, Nicosia, Turkey.

4 Faculty of Engineering and Natural Sciences, Bahcesehir University, Istanbul, Turkey.

*Corresponding author: ali.ahmadian@ukm.edu.my 
than T1FSs. The most largely used T2FSs are "interval type-2 fuzzy sets" (IT2FSs) [26-28] that is a particular case of frequently used T2FSs.

"Intuitionistic fuzzy sets" (IFSs) are launched by Atanassov [2] that consider both membership and nonmembership grades. Many investigators have declared their uniqueness in decision-making because of their significance in dealing with uncertainty. To depict the indeterminacy membership grade, IFSs were extended by Smarandache [35] to "neutrosophic sets" (NSs) characterized by "truth membership function" (TMF), "indeterminacy membership function" (IMF) and "falsity membership function" (FMF). Subsequently, the thought of "single-valued neutrosophic set" (SVNS) [43] was presented to cope up with vague, indefinite and incoherent facts and figures. "Interval type-2 neutrosophic sets" (IT2NSs) [41,53] were launched to determine the improbability and haziness more proficiently.

In literature, there are various MCDM techniques including ELECTRE, fuzzy TOPSIS, fuzzy VIKOR, PROMETHEE, fuzzy AHP, fuzzy ANP and so on [8,9,12,22]. Peng et al. [30] presented an expansion of ELECTRE for solving MCDM setbacks where the weights and other information was depicted by using multihesitant FSs. Shaygan et al. [34] anticipated a tactic formed on fuzzy AHP for decision-making to classify, categorize and pick schemes. Akram et al. [1] also presented an approach by extending the TOPSIS method for MCDM problems using soft sets. Gupta et al. [21] expanded the TOPSIS technique with interval-valued IFSs to solve MCDM problems. Touqeer et al. [38-40] also presented various techniques for solving MCDM problems in fuzzy environment using different types of fuzzy numbers. All these MCDM techniques utilizes FSs that are not capable of handling indeterminacy and irregularity involved during these processes so, in the recent times, some neutrosophic MCDM approaches have been creatively established to handle vagueness, indeterminacy and inconsistency in decision-making problems including PROMETHEE method for MCDM problems based on probability multi-valued NSs extended by Liu et al. [23]. Biswas et al. [5] presented a value and ambiguity-based ranking approach for trapezoidal neutrosophic number (TrNNs) and presented an MCDM strategy. Deli et al. [17] proposed a ranking strategy of TrNN for solving MCDM problems. Liang et al. [24] defined score, accuracy and certainty function in the context of single-valued trapezoidal neutrosophic number (SVTrNN) by using the concept of center of gravity (COG). Biswas et al. [7] presented TOPSIS approach for MCDM in TrNN atmosphere. Pramanik et al. [31] established a VIKOR method for a MCDM in TrNN framework. Giri et al. [20] suggested an MCDM method involving interval trapezoidal neutrosophic number (ITrNN). Pramanik et al. [32] proposed TODIM strategy for MCDM in TrNN environment.

One more approach involving MCDM problem under fuzzy framework was presented by Yang et al. [51] where TOPSIS is extended in trapezoidal interval type-2 fuzzy environment using $\alpha$-cuts. As FSs plays no role in indeterminacy values and non-membership functions and are only engaged with membership function so, they are not able to cope up with the vagueness and non-deterministic situations occurring during the MCDM process hence, in this research, we have extended this approach in neutrosophic environment. We propose an extended TOPSIS approach where the OWA operator is used to reflect the attitudes and perspectives of decision-makers. The core difference is that the presented approach utilizes interval type-2 neutrosophic numbers (IT2NNs) and uses the tool of $(\alpha, \beta, \gamma)$-cut to deneutrosify the IT2NNs. Based on IT2NN structure, this paper utilizes a wellknown neutrosophic number having trapezoidal appearance called an "interval type-2 trapezoidal neutrosophic number" (IT2TrNN) [42].

The idea of this article is to establish an inventive and effective method for dealing with MCDM problems under IT2TrNN framework. The IT2TrNNs are used to articulate the performance ratings of criterion in MCDM problems as they can proficiently convey qualitative assessments of decision-makers. Moreover, $(\alpha, \beta, \gamma)$ cut approach is used to compute the distance between two IT2TrNNs. By using the tool of $(\alpha, \beta, \gamma)$-cut, the constraints concerned with the shapes of neutrosophic sets can be avoided and it can lessen the effect of uncertainty inherent in MCDM problems and provide us with more accurate solution. Also, an integral operation of $(\alpha, \beta, \gamma)$-cut is used to calculate the distance between two neutrosophic numbers. Furthermore, OWA operator is used to determine the distance between two IT2TrNNs as it can depict the outlook of decision-makers. Finally, a constructive and precise analytical solution of distance between two IT2TrNNs is derived formed on our 
projected distance approach, which can serve as a uncomplicated and exact distance manner for many applications in the neutrosophic systems.

The rest of the article is ordered as follows: Section 2 recalls some fundamental concepts about IT2NNs, $(\alpha, \beta, \gamma)$-cut, OWA operator and classical TOPSIS technique. Section 3 presents a technique to compute distance between two IT2TrNNs using the notion of $(\alpha, \beta, \gamma)$-cut and OWA operator and also projects an analytical solution of the distance between two IT2TrNNs. Section 4 anticipates a detailed methodology of extension of TOPSIS in the context of IT2TrNNs using the projected distance approach. Section 5 demonstrates an numerical example to prove the feasibility of the proposed approach. Section 6 compares the proposed technique with previous techniques to demonstrate the viability of presented approach. Section 7 sums up the paper and provides conclusion.

\section{Preliminaries}

Several related definitions and concepts about interval type- 2 neutrosophic numbers, $(\alpha, \beta, \gamma)$-cut, OWA operator and TOPSIS method used in the subsequent discussions are briefly reviewed in the following section.

\subsection{Interval type-2 neutrosophic numbers}

Definition 2.1 ([35,36]). A single-valued neutrosophic set (SVNS) $\breve{S}$ on universal set $\mathbf{U}$ is characterized by $\operatorname{TMF}\left(\phi_{\breve{\mathrm{S}}}\right), \operatorname{IMF}\left(\psi_{\breve{\mathrm{S}}}\right)$ and $\operatorname{FMF}\left(\varphi_{\breve{\mathrm{S}}}\right)$ respectively, in the following way:

$$
\breve{S}=\left\{\left\langle\xi,\left(\phi_{\breve{S}}(\xi), \psi_{\breve{S}}(\xi), \varphi_{\breve{S}}(\xi)\right)\right\rangle: \xi \in \mathbf{U}, \phi_{\breve{S}}(\xi), \psi_{\breve{S}}(\xi), \varphi_{\breve{S}}(\xi) \in[0,1]\right\}
$$

such that $0 \leq \phi_{\breve{S}}(\xi), \psi_{\breve{S}}(\xi), \varphi_{\breve{S}}(\xi) \leq 3$.

Definition 2.2 ([42]). Let $\breve{S}(\xi)=\left[\breve{S}^{U}(\xi), \breve{S}^{L}(\xi)\right]$ be an interval type-2 neutrosophic set (IT2NS) on universal set $\mathbf{U}$ where $\xi \in \mathbf{U}$ and $\breve{\mathrm{S}}^{U}: \mathbf{U} \rightarrow[0,1]$ and $\breve{\mathrm{S}}^{L}: \mathbf{U} \rightarrow[0,1]$ are two type-1 neutrosophic sets (T1NSs) known as upper and lower neutrosophic sets respectively having the condition $0 \leq \breve{\mathrm{S}}^{L}(\xi) \leq \breve{\mathrm{S}}^{U}(\xi) \leq 1$ defined as follows:

$$
\begin{aligned}
\breve{\mathrm{S}}=\{ & \left\{\left\langle\xi,\left(\left[\phi_{\breve{\mathrm{S}}}^{U}(\xi), \phi_{\breve{\mathrm{S}}}^{L}(\xi)\right],\left[\psi_{\breve{\mathrm{S}}}^{U}(\xi), \psi_{\breve{\mathrm{S}}}^{L}(\xi)\right],\left[\varphi_{\breve{\mathrm{S}}}^{U}(\xi), \varphi_{\breve{\mathrm{S}}}^{L}(\xi)\right]\right)\right\rangle: \xi \in \mathbf{U},\right. \\
& {\left.\left[\phi_{\breve{\mathrm{S}}}^{U}(\xi), \phi_{\breve{\mathrm{S}}}^{L}(\xi)\right],\left[\psi_{\breve{\mathrm{S}}}^{U}(\xi), \psi_{\breve{\mathrm{S}}}^{L}(\xi)\right],\left[\varphi_{\breve{\mathrm{S}}}^{U}(\xi), \varphi_{\breve{\mathrm{S}}}^{L}(\xi)\right] \in[0,1]\right\} . }
\end{aligned}
$$

Definition $2.3([42])$. Let $\breve{S}$ be a trapezoidal neutrosophic number $(\operatorname{TrNN})$ with $\operatorname{TMF}\left(\phi_{\breve{S}}\right)$, IMF $\left(\psi_{\breve{S}}\right)$ and $\operatorname{FMF}\left(\varphi_{\breve{S}}\right)$ defined as follows:

$$
\begin{aligned}
& \phi_{\breve{\mathrm{S}}}(\xi)= \begin{cases}\frac{\left(\xi-\breve{\mathrm{s}}_{1}\right) \phi_{\breve{\mathrm{S}}}}{\breve{\mathrm{s}}_{2}-\breve{\mathrm{s}}_{1}} & \breve{\mathrm{s}}_{1} \leq \xi<\breve{\mathrm{s}}_{2} \\
\phi_{\breve{\mathrm{S}}} & \breve{\mathrm{s}}_{2} \leq \xi \leq \breve{\mathrm{s}}_{3} \\
\frac{\left(\breve{\mathrm{s}}_{4}-\xi\right) \phi_{\breve{\mathrm{S}}}}{\breve{\mathrm{s}}_{4}-\breve{\mathrm{s}}_{3}} & \breve{\mathrm{S}}_{3}<\xi \leq \breve{\mathrm{s}}_{4} \\
0 & \text { otherwise }\end{cases}
\end{aligned}
$$

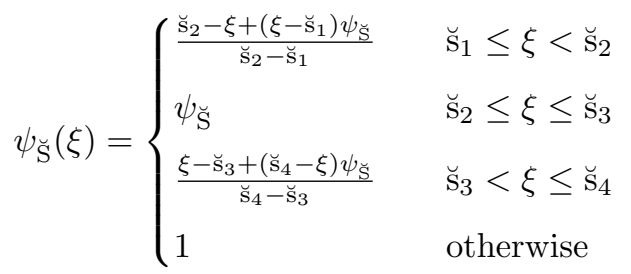




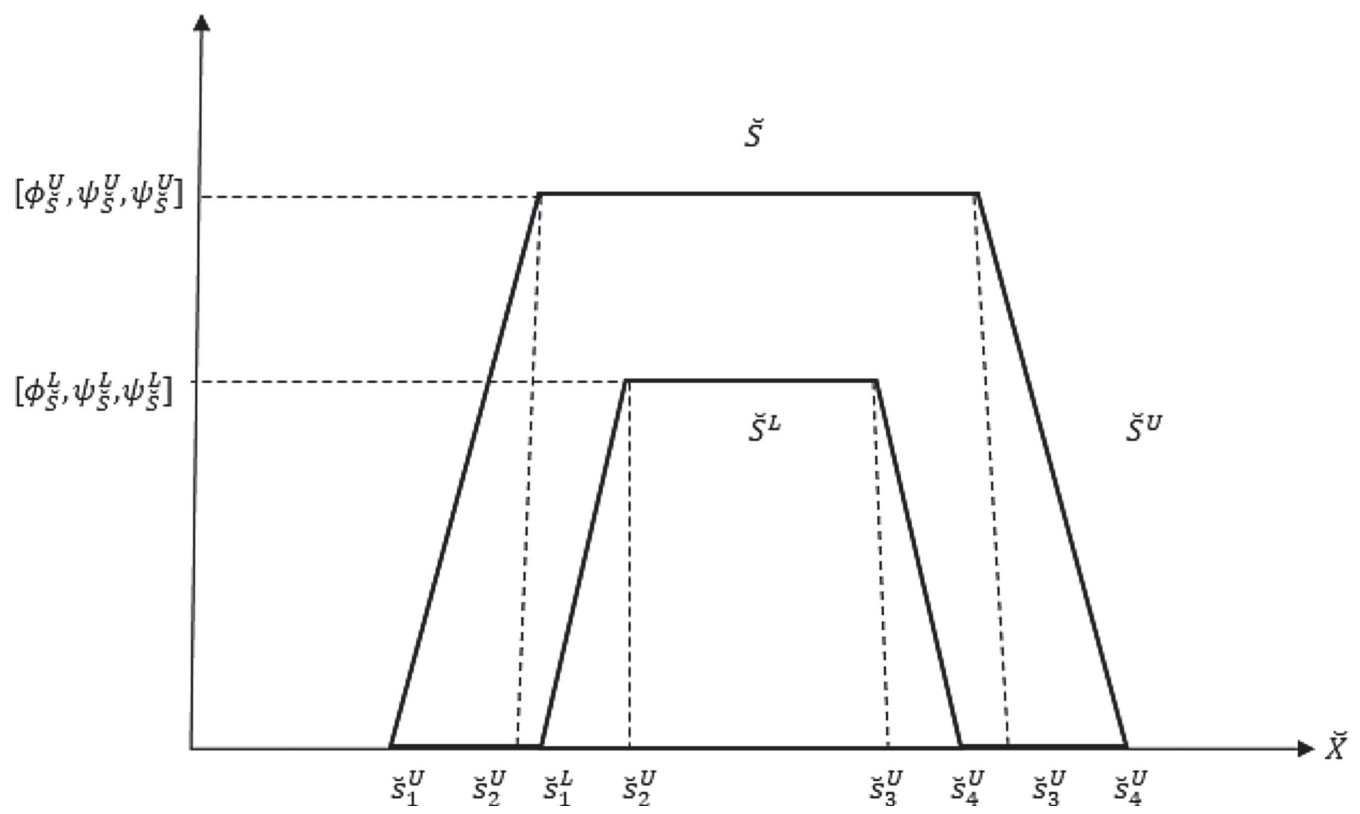

FIGURE 1. An interval type-2 trapezoidal neutrosophic number.

$$
\varphi_{\breve{\mathrm{S}}}(\xi)= \begin{cases}\frac{\breve{\mathrm{s}}_{2}-\xi+\left(\xi-\breve{\mathrm{s}}_{1}\right) \varphi_{\breve{\mathrm{S}}}}{\breve{\mathrm{s}}_{2}-\breve{\mathrm{s}}_{1}} & \breve{\mathrm{s}}_{1} \leq \xi<\breve{\mathrm{s}}_{2} \\ \varphi_{\breve{\mathrm{S}}} & \breve{\mathrm{s}}_{2} \leq \xi \leq \breve{\mathrm{s}}_{3} \\ \frac{\xi-\breve{\mathrm{s}}_{3}+\left(\breve{\mathrm{s}}_{4}-\xi\right) \varphi_{\breve{S}}}{\breve{\mathrm{s}}_{4}-\breve{\mathrm{s}}_{3}} & \breve{\mathrm{s}}_{3}<\xi \leq \breve{\mathrm{s}}_{4} \\ 1 & \text { otherwise }\end{cases}
$$

where $\phi_{\breve{S}}=\left[\phi_{\breve{S}}^{U}, \phi_{\breve{S}}^{L}\right], \psi_{\breve{S}}=\left[\psi_{\breve{S}}^{U}, \psi_{\breve{S}}^{L}\right]$ and $\varphi_{\breve{S}}=\left[\varphi_{\breve{S}}^{U}, \varphi_{\breve{S}}^{L}\right]$ are interval type-2 neutrosophic numbers (IT2NNs).

The number $\breve{\mathrm{S}}$ can be represented as

$$
\breve{\mathrm{S}}=\left[\breve{\mathrm{S}}^{U}, \breve{\mathrm{S}}^{L}\right]=\left[\left(\breve{\mathrm{S}}_{1}^{U}, \breve{\mathrm{s}}_{2}^{U}, \breve{\mathrm{s}}_{3}^{U}, \breve{\mathrm{s}}_{4}^{U} ; \phi_{\breve{\mathrm{S}}}^{U}, \psi_{\breve{\mathrm{S}}}^{U}, \varphi_{\breve{\mathrm{S}}}^{U}\right),\left(\breve{\mathrm{S}}_{1}^{L}, \breve{\mathrm{s}}_{2}^{L}, \breve{\mathrm{s}}_{3}^{L}, \breve{\mathrm{s}}_{4}^{L} ; \phi_{\breve{\mathrm{S}}}^{L}, \psi_{\breve{\mathrm{S}}}^{L}, \varphi_{\breve{\mathrm{S}}}^{L}\right)\right]
$$

and is called interval type-2 trapezoidal neutrosophic number (IT2TrNN) where $0 \leq \breve{\mathrm{s}}_{1}^{U} \leq \breve{\mathrm{s}}_{2}^{U} \leq \breve{\mathrm{s}}_{3}^{U} \leq \breve{\mathrm{s}}_{4}^{U} \leq 1$, $0 \leq \breve{\mathrm{S}}_{1}^{L} \leq \breve{\mathrm{s}}_{2}^{L} \leq \breve{\mathrm{s}}_{3}^{L} \leq \breve{\mathrm{s}}_{4}^{L} \leq 1,0 \leq \phi_{\breve{\mathrm{S}}}^{L} \leq \phi_{\breve{\mathrm{S}}}^{U} \leq 1,0 \leq \psi_{\breve{\mathrm{S}}}^{L} \leq \psi_{\breve{\mathrm{S}}}^{U} \leq 1$ and $0 \leq \varphi_{\breve{\mathrm{S}}}^{L} \leq \varphi_{\breve{\mathrm{S}}}^{U} \leq 1$ (Fig. 1).

Definition 2.4. Let $\breve{\mathrm{S}}=\left(\breve{\mathrm{S}}^{U}, \breve{\mathrm{S}}^{L}\right)=\left[\left(\breve{\mathrm{s}}_{1}^{U}, \breve{\mathrm{s}}_{2}^{U}, \breve{\mathrm{s}}_{3}^{U}, \breve{\mathrm{s}}_{4}^{U} ; \phi_{\breve{\mathrm{S}}}^{U}, \psi_{\breve{\mathrm{S}}}^{U}, \varphi_{\breve{\mathrm{S}}}^{U}\right),\left(\breve{\mathrm{s}}_{1}^{L}, \breve{s}_{2}^{L}, \breve{\mathrm{s}}_{3}^{L}, \breve{\mathrm{s}}_{4}^{L} ; \phi_{\breve{\mathrm{S}}}^{L}, \psi_{\breve{\mathrm{S}}}^{L}, \varphi_{\breve{\mathrm{S}}}^{L}\right)\right]$ and $\breve{\mathrm{T}}=$ $\left(\breve{\mathrm{T}}^{U}, \breve{\mathrm{T}}^{L}\right)=\left[\left(\breve{\mathrm{t}}_{1}^{U}, \breve{\mathrm{t}}_{2}^{U}, \breve{\mathrm{t}}_{3}^{U}, \breve{\mathrm{t}}_{4}^{U} ; \phi_{\breve{\mathrm{T}}}^{U}, \psi_{\breve{\mathrm{T}}}^{U}, \varphi_{\breve{\mathrm{T}}}^{U}\right),\left(\breve{\mathrm{t}}_{1}^{L}, \breve{\mathrm{t}}_{2}^{L}, \breve{\mathrm{t}}_{3}^{L}, \breve{\mathrm{t}}_{4}^{L} ; \phi_{\breve{\mathrm{T}}}^{L}, \psi_{\breve{\mathrm{T}}}^{L}, \varphi_{\breve{\mathrm{T}}}^{L}\right)\right]$ be two IT2TrNNs. We introduce the arithmetic operations of addition and subtraction on $\breve{\mathrm{S}}$ and $\breve{\mathrm{T}}$ defined as follows:

(i) Addition:

$$
\begin{aligned}
\breve{\mathrm{S}} \oplus \breve{\mathrm{T}}= & \left(\left[\breve{\mathrm{s}}_{1}^{U}+\breve{\mathrm{t}}_{1}^{U}, \breve{\mathrm{s}}_{2}^{U}+\breve{\mathrm{t}}_{2}^{U}, \breve{\mathrm{s}}_{3}^{U}+\breve{\mathrm{t}}_{3}^{U}, \breve{\mathrm{s}}_{4}^{U}+\breve{\mathrm{t}}_{4}^{U} ; \min \left(\left(\phi_{\breve{\mathrm{S}}}^{U}, \psi_{\breve{\mathrm{S}}}^{U}, \varphi_{\breve{\mathrm{S}}}^{U}\right),\left(\phi_{\breve{\mathrm{T}}}^{U}, \psi_{\breve{\mathrm{T}}}^{U}, \varphi_{\breve{\mathrm{T}}}^{U}\right)\right)\right],\right. \\
& {\left.\left[\breve{\mathrm{s}}_{1}^{L}+\breve{\mathrm{t}}_{1}^{L}, \breve{\mathrm{s}}_{2}^{L}+\breve{\mathrm{t}}_{2}^{L}, \breve{\mathrm{s}}_{3}^{L}+\breve{\mathrm{t}}_{3}^{L}, \breve{\mathrm{s}}_{4}^{L}+\breve{\mathrm{t}}_{4}^{L} ; \min \left(\left(\phi_{\breve{\mathrm{S}}}^{L}, \psi_{\breve{\mathrm{S}}}^{L}, \varphi_{\breve{\mathrm{S}}}^{L}\right),\left(\phi_{\breve{\mathrm{T}}}^{L}, \psi_{\breve{\mathrm{T}}}^{L}, \varphi_{\breve{\mathrm{T}}}^{L}\right)\right)\right]\right) . }
\end{aligned}
$$


(ii) Subtraction:

$$
\begin{aligned}
\breve{\mathrm{S}}-\breve{\mathrm{T}}= & \left(\left[\breve{\mathrm{s}}_{1}^{U}-\breve{\mathrm{t}}_{4}^{U}, \breve{\mathrm{s}}_{2}^{U}-\breve{\mathrm{t}}_{3}^{U}, \breve{\mathrm{s}}_{3}^{U}-\breve{\mathrm{t}}_{2}^{U}, \breve{\mathrm{s}}_{4}^{U}-\breve{\mathrm{t}}_{1}^{U} ; \min \left(\left(\phi_{\breve{\mathrm{S}}}^{U}, \psi_{\breve{\mathrm{S}}}^{U}, \varphi_{\breve{\mathrm{S}}}^{U}\right),\left(\phi_{\breve{\mathrm{T}}}^{U}, \psi_{\breve{\mathrm{T}}}^{U}, \varphi_{\breve{\mathrm{T}}}^{U}\right)\right)\right],\right. \\
& {\left.\left[\breve{\mathrm{s}}_{1}^{L}-\breve{\mathrm{t}}_{4}^{L}, \breve{\mathrm{s}}_{2}^{L}-\breve{\mathrm{t}}_{3}^{L}, \breve{\mathrm{s}}_{3}^{L}-\breve{\mathrm{t}}_{2}^{L}, \breve{\mathrm{s}}_{4}^{L}-\breve{\mathrm{t}}_{1}^{L} ; \min \left(\left(\phi_{\breve{\mathrm{S}}}^{L}, \psi_{\breve{\mathrm{S}}}^{L}, \varphi_{\breve{\mathrm{S}}}^{L}\right),\left(\phi_{\breve{\mathrm{T}}}^{L}, \psi_{\breve{\mathrm{T}}}^{L}, \varphi_{\breve{\mathrm{T}}}^{L}\right)\right)\right]\right) . }
\end{aligned}
$$

\section{2. $(\alpha, \beta, \gamma)$-cut}

Definition 2.5 ([37]). The $(\alpha, \beta, \gamma)$-cut of a neutrosophic number denoted by $\left.\breve{\mathrm{S}}_{(} \alpha, \beta, \gamma\right)$ is defined as:

$$
\breve{\mathrm{S}}_{(\alpha, \beta, \gamma)}=\left\{\left\langle\xi,\left(\phi_{\breve{\mathrm{S}}}(\xi), \psi_{\breve{\mathrm{S}}}(\xi), \varphi_{\breve{\mathrm{S}}}(\xi)\right)\right\rangle: \xi \in \mathbf{U}, \phi_{\breve{\mathrm{S}}}(\xi) \geq \alpha, \psi_{\breve{\mathrm{S}}}(\xi) \leq \beta, \varphi_{\breve{\mathrm{S}}}(\xi) \leq \gamma\right\}
$$

where $\alpha, \beta, \gamma \in[0,1]$ and are fixed numbers such that $\alpha+\beta+\gamma \leq 3$.

Definition 2.6 ([37]). The $(\alpha, \beta, \gamma)$-cut of an IT2NN $\breve{S}$ is represented as follows:

$$
\begin{aligned}
\breve{\mathrm{S}} & =\left\{\left\langle\xi,\left(\left[\phi_{\breve{\mathrm{S}}}^{U}(\xi), \phi_{\breve{\mathrm{S}}}^{L}(\xi)\right] \geq \alpha,\left[\psi_{\breve{\mathrm{S}}}^{U}(\xi), \psi_{\breve{\mathrm{S}}}^{L}(\xi)\right] \leq \beta,\left[\varphi_{\breve{\mathrm{S}}}^{U}(\xi), \varphi_{\breve{\mathrm{S}}}^{L}(\xi)\right] \leq \gamma\right)\right\rangle\right\} \\
& =[\breve{\mathrm{s}}(\alpha), \breve{\mathrm{t}}(\alpha)],[\breve{\mathrm{s}}(\beta), \breve{\mathrm{t}}(\beta)],[\breve{\mathrm{s}}(\gamma), \breve{\mathrm{t}}(\gamma)]
\end{aligned}
$$

where $\xi \in \mathbf{U},\left[\phi_{\breve{\mathrm{S}}}^{U}(\xi), \phi_{\breve{\mathrm{S}}}^{L}(\xi)\right],\left[\psi_{\breve{\mathrm{S}}}^{U}(\xi), \psi_{\breve{\mathrm{S}}}^{L}(\xi)\right],\left[\varphi_{\breve{\mathrm{S}}}^{U}(\xi), \varphi_{\breve{\mathrm{S}}}^{L}(\xi)\right], \alpha, \beta, \gamma \in[0,1]$ and $\breve{\mathrm{s}}(\alpha) \in\left[\breve{\mathrm{s}}_{l}(\alpha), \breve{\mathrm{s}}_{r}(\alpha)\right], \breve{\mathrm{t}}(\alpha) \in$ $\left[\breve{\mathrm{t}}_{l}(\alpha), \breve{\mathrm{t}}_{r}(\alpha)\right], \breve{\mathrm{s}}(\beta) \in\left[\breve{\mathrm{s}}_{l}(\beta), \breve{\mathrm{s}}_{r}(\beta)\right], \breve{\mathrm{t}}(\beta) \in\left[\breve{\mathrm{t}}_{l}(\beta), \breve{\mathrm{t}}_{r}(\beta)\right], \breve{\mathrm{s}}(\gamma) \in\left[\breve{\mathrm{s}}_{l}(\gamma), \breve{\mathrm{s}}_{r}(\gamma)\right], \breve{\mathrm{t}}(\gamma) \in\left[\breve{\mathrm{t}}_{l}(\gamma), \breve{\mathrm{t}}_{r}(\gamma)\right]$.

If $(\alpha, \beta, \gamma)$-cut on $\breve{\mathrm{S}}^{L}$ exists, then the intervals $[\breve{\mathrm{s}}(\alpha), \breve{\mathrm{t}}(\alpha)],[\breve{\mathrm{s}}(\beta), \breve{\mathrm{t}}(\beta)],[\breve{\mathrm{s}}(\gamma), \breve{\mathrm{t}}(\gamma)]$ are divided into

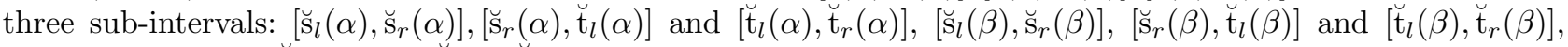
$\left[\breve{\mathrm{s}}_{l}(\gamma), \breve{\mathrm{s}}_{r}(\gamma)\right],\left[\breve{\mathrm{s}}_{r}(\gamma), \breve{\mathrm{t}}_{l}(\gamma)\right]$ and $\left[\breve{\mathrm{t}}_{l}(\gamma), \breve{\mathrm{t}}_{r}(\gamma)\right]$ respectively.

$\breve{\mathrm{s}}(\alpha), \breve{\mathrm{s}}(\beta)$ and $\breve{\mathrm{s}}(\gamma)$ cannot assume a value larger than $\breve{\mathrm{s}}_{r}(\alpha), \breve{\mathrm{s}}_{r}(\beta)$ and $\breve{\mathrm{s}}_{r}(\gamma)$. Similarly, $\breve{\mathrm{t}}(\alpha) \in$ $\left[\breve{\mathrm{t}}_{l}(\alpha), \breve{\mathrm{t}}_{r}(\alpha)\right], \breve{\mathrm{t}}(\beta) \in\left[\breve{\mathrm{t}}_{l}(\beta), \breve{\mathrm{t}}_{r}(\beta)\right]$ and $\breve{\mathrm{t}}(\gamma) \in\left[\breve{\mathrm{t}}_{l}(\gamma), \breve{\mathrm{t}}_{r}(\gamma)\right]$ cannot assume a value smaller than $\breve{\mathrm{t}}_{l}(\alpha), \breve{\mathrm{t}}_{l}(\beta)$ and $\breve{\mathrm{t}}_{l}(\gamma)$ respectively. However, if $(\alpha, \beta, \gamma)$-cut on $\breve{\mathrm{S}}^{L}$ doesn't exist, then both $\breve{\mathrm{s}}_{r}(\alpha)$ and $\breve{\mathrm{t}}_{l}(\alpha)$, $\breve{\mathrm{s}}_{r}(\beta)$ and $\breve{\mathrm{t}}_{l}(\beta)$ and $\breve{\mathrm{s}}_{r}(\gamma)$ and $\breve{\mathrm{t}}_{l}(\gamma)$ can assume values freely in the entire intervals $\left[\breve{\mathrm{s}}_{l}(\alpha), \breve{\mathrm{t}}_{r}(\alpha)\right],\left[\breve{\mathrm{s}}_{l}(\beta), \breve{\mathrm{t}}_{r}(\beta)\right]$ and $\left[\breve{\mathrm{s}}_{l}(\gamma), \breve{\mathrm{t}}_{r}(\gamma)\right]$ (Fig. 2).

$$
\begin{aligned}
& \breve{\mathrm{s}}(\alpha) \in \begin{cases}{\left[\breve{\mathrm{s}}_{l}(\alpha), \breve{\mathrm{s}}_{r}(\alpha)\right]} & \alpha \in\left[0, \phi_{\breve{\mathrm{S}}}^{L}\right] \\
{\left[\breve{\mathrm{s}}_{l}(\alpha), \breve{\mathrm{t}}_{r}(\alpha)\right]} & \alpha \in\left[\phi_{\breve{\mathrm{S}}}^{L}, 1\right]\end{cases} \\
& \breve{\mathrm{t}}(\alpha) \in \begin{cases}{\left[\breve{\mathrm{t}}_{l}(\alpha), \breve{\mathrm{t}}_{r}(\alpha)\right]} & \alpha \in\left[0, \phi_{\breve{\mathrm{s}}}^{L}\right] \\
{\left[\breve{\mathrm{s}}_{l}(\alpha), \breve{\mathrm{t}}_{r}(\alpha)\right]} & \alpha \in\left[\phi_{\breve{\mathrm{S}}}^{L}, 1\right]\end{cases} \\
& \breve{\mathrm{s}}(\beta) \in \begin{cases}{\left[\breve{\mathrm{s}}_{l}(\beta), \breve{\mathrm{s}}_{r}(\beta)\right]} & \beta \in\left[0, \psi_{\breve{\mathrm{s}}}^{L}\right] \\
{\left[\breve{\mathrm{s}}_{l}(\beta), \breve{\mathrm{t}}_{r}(\beta)\right]} & \beta \in\left[\psi_{\breve{\mathrm{S}}}^{L}, 1\right]\end{cases} \\
& \breve{\mathrm{t}}(\beta) \in \begin{cases}{\left[\breve{\mathrm{t}}_{l}(\beta), \breve{\mathrm{t}}_{r}(\beta)\right]} & \beta \in\left[0, \psi_{\breve{\mathrm{s}}}^{L}\right] \\
{\left[\breve{\mathrm{s}}_{l}(\beta), \breve{\mathrm{t}}_{r}(\beta)\right]} & \beta \in\left[\psi_{\breve{\mathrm{S}}}^{L}, 1\right]\end{cases} \\
& \breve{\mathrm{s}}(\gamma) \in \begin{cases}{\left[\breve{\mathrm{s}}_{l}(\gamma), \breve{\mathrm{s}}_{r}(\gamma)\right]} & \gamma \in\left[0, \varphi_{\breve{\mathrm{s}}}^{L}\right] \\
{\left[\breve{\mathrm{s}}_{l}(\gamma), \breve{\mathrm{t}}_{r}(\gamma)\right]} & \gamma \in\left[\varphi_{\breve{\mathrm{S}}}^{L}, 1\right]\end{cases}
\end{aligned}
$$




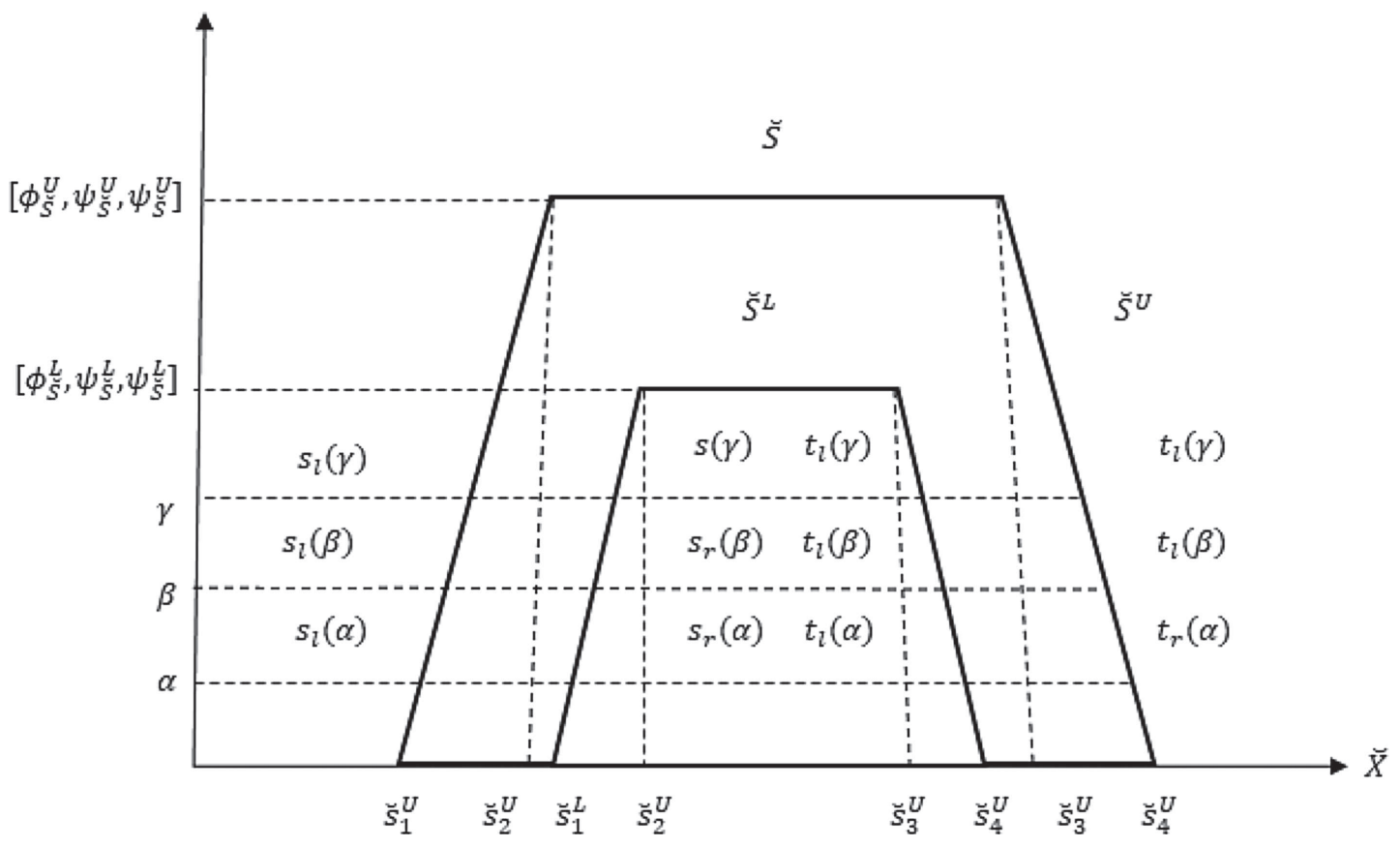

Figure 2. The $(\alpha, \beta, \gamma)$-cut of an IT2TrNN.

$$
\breve{\mathrm{t}}(\gamma) \in \begin{cases}{\left[\breve{\mathrm{t}}_{l}(\gamma), \breve{\mathrm{t}}_{r}(\gamma)\right]} & \gamma \in\left[0, \varphi_{\breve{\mathrm{S}}}^{L}\right] \\ {\left[\breve{\mathrm{s}}_{l}(\gamma), \breve{\mathrm{t}}_{r}(\gamma)\right]} & \gamma \in\left[\varphi_{\breve{\mathrm{S}}}^{L}, 1\right] .\end{cases}
$$

\subsection{OWA operator}

Definition 2.7 ([33]). An OWA operator having dimension $n$ is a mapping $\breve{\mathrm{S}}: \mathbf{R}^{n} \rightarrow \mathbf{R}$ associated with an $n$ vector $\breve{\mathrm{W}}=\left(\breve{\mathrm{w}}_{1}, \ldots, \breve{\mathrm{w}}_{n}\right)$ such that $\breve{\mathrm{w}}_{i} \in[0,1]$ and $\sum_{i=1}^{n} \breve{\mathrm{w}}_{i}=1$. Moreover,

$$
\breve{\mathrm{S}}_{\breve{\mathrm{w}}}\left(\breve{\mathrm{S}}_{1}, \ldots, \breve{\mathrm{s}}_{n}\right)=\sum_{j=1}^{n} \breve{\mathrm{w}}_{j} \breve{\mathrm{t}}_{j}
$$

where $\breve{\mathrm{t}}_{j}$ represents $j$-th largest element of aggregated objects collection $\breve{s}_{1}, \ldots, \breve{\mathrm{s}}_{n}$.

\subsection{TOPSIS method}

The "Technique for Order Preference by Similarity to Ideal Solution" (TOPSIS) is the most recognized techniques to deal with MCDM setbacks in different application fields and the primary foundation of TOPSIS is that the optimal alternative has shortest distance from "Positive Ideal Solution" (PIS) and farthest distance from "Negative Ideal Solution" (NIS). Assuming a MCDM problem having $n$ alternatives $\left(\mathrm{L}_{1}, \ldots, \mathrm{L}_{n}\right)$ and $m$ criterion $\left(\mathrm{R}_{1}, \ldots, \mathrm{R}_{n}\right)$. Each alternative is to be evaluated according to the $n$ criterion. All the values that are assigned to the alternative for each criterion are shown by the decision matrix $\mathrm{X}=\left(x_{i j}\right)_{n \times m}$. $\breve{\mathrm{W}}=\left(\breve{\mathrm{w}}_{1}, \ldots, \breve{\mathrm{w}}_{m}\right)$ shows the criterion weights satisfying $\sum_{j=1}^{m} \breve{\mathrm{w}}_{j}=1$. 


\subsubsection{Algorithm of TOPSIS}

Step 1. Build a normalized decision matrix.

For benefit type criteria:

$$
\mathrm{n}_{i j}=\frac{x_{i j}}{\max \left(x_{i j}\right)}
$$

For cost type criteria:

$$
\mathrm{n}_{i j}=\frac{\min \left(x_{i j}\right)}{x_{i j}}
$$

where $\mathrm{n}_{i j}$ is the normalized value of $x_{i j}$.

Step 2. Compute weighted normalized decision matrix $\mathrm{V}=\left(v_{i j}\right)_{n \times m}$.

$$
v_{i j}=\breve{\mathrm{w}}_{j} \mathrm{n}_{i j}
$$

where $\breve{\mathrm{w}}_{j}$ is the $j$-th criterion weight and $\sum_{j=1}^{m} \breve{\mathrm{w}}_{j}=1$.

Step 3. Evaluate the PIS and NIS.

$$
\begin{aligned}
\mathrm{P}^{*} & =\left\{v_{1}^{*}, v_{2}^{*}, \ldots, v_{n}^{*}\right\} \\
& =\left\{\left(\max _{i} v_{i j} \mid j \in \mathrm{K}_{t}\right)\left(\min _{i} v_{i j} \mid j \in \mathrm{K}_{c}\right)\right\} \\
\mathrm{N}^{-} & =\left\{v_{1}^{-}, v_{2}^{-}, \ldots, v_{n}^{-}\right\} \\
& =\left\{\left(\max _{i} v_{i j} \mid j \in \mathrm{K}_{c}\right)\left(\min _{i} v_{i j} \mid j \in \mathrm{K}_{t}\right)\right\}
\end{aligned}
$$

where $\mathrm{K}_{t}$ is the benefit criterion set and $\mathrm{K}_{c}$ is the cost criterion set.

Step 4. Acquire the distances of alternatives from PIS and NIS.

$$
\begin{aligned}
\mathrm{D}_{i}^{*} & =\sqrt{\sum_{j=1}^{n}\left(v_{i j}-v_{j}^{*}\right)^{2}} \\
\mathrm{D}_{i}^{-} & =\sqrt{\sum_{j=1}^{n}\left(v_{i j}-v_{j}^{-}\right)^{2}} .
\end{aligned}
$$

Step 5. Compute relative closeness to the ideal alternatives.

$$
\mathrm{RC}_{i}=\frac{\mathrm{D}_{i}^{-}}{\mathrm{D}_{i}^{-}+\mathrm{D}_{i}^{*}}
$$

Step 6. Rank the alternatives with respect to the relative closeness to the ideal alternatives. The larger the relative closeness coefficient, is the better alternative.

\section{Method to compute distance Between two IT2TrNns}

In the following segment, we projected a new technique to compute the distance between two IT2TrNNs by using the conception of $(\alpha, \beta, \gamma)$-cuts and OWA operator that can be used to determine the distances from an alternative to PIS and NIS whose ratings of local criterion are represented in the form of IT2TrNNs. Moreover, we established an analytical solution of the distance between two IT2TrNNs that can be used for calculating the distance more suitably and easily.

The techniques of computing the distance between two IT2TrNNs are shown as follows: 


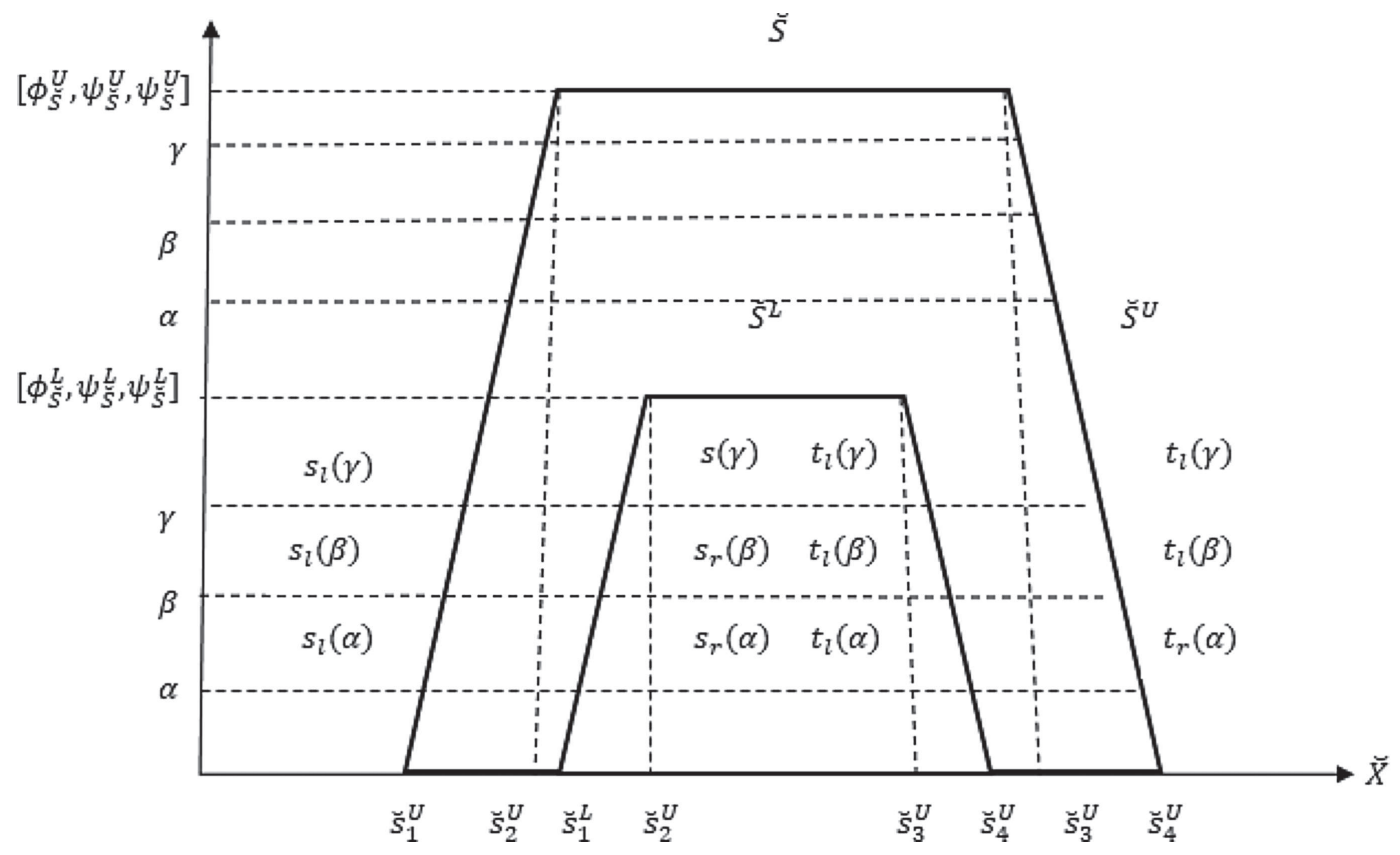

FiguRE 3. The $(\alpha, \beta, \gamma)$-cut of an IT2TrNN.

Firstly, a new assumption is made about the $(\alpha, \beta, \gamma)$-cuts of a IT2TrNN when $\alpha, \beta$ and $\gamma$ are over the lower truth, indeterminacy and falsity membership functions i.e. $\phi_{\breve{S}}^{L}, \psi_{\breve{S}}^{L}$ and $\varphi_{\breve{S}}^{L}$. For a IT2TrNN $\breve{S}$ (seen in Fig. 3), it is assumed that when $\alpha, \beta, \gamma>\phi_{\breve{\mathrm{S}}}^{L}, \psi_{\breve{\mathrm{S}}}^{L}, \varphi_{\breve{\mathrm{S}}}^{L}, \breve{\mathrm{s}}(\alpha)$ values freely in the interval [ $\left.\breve{\mathrm{s}}_{l}(\alpha), \breve{\mathrm{t}}_{r}(\alpha)\right]$ and $\breve{\mathrm{t}}(\alpha)$ also values freely in the interval $\left[\breve{\mathrm{s}}_{l}(\alpha), \breve{\mathrm{t}}_{r}(\alpha)\right]$, which means the $\alpha$-cuts of a IT2TrNN are $\left[\left(\left[\breve{\mathrm{s}}_{l}(\alpha), \breve{\mathrm{t}}_{r}(\alpha)\right]\right),\left(\left[\breve{\mathrm{s}}_{l}(\alpha), \breve{\mathrm{t}}_{r}(\alpha)\right]\right)\right]$ when $\alpha>\phi_{\breve{\mathrm{s}}}^{L}$. Similarly, $\breve{\mathrm{s}}(\beta)$ values freely in the interval $\left[\breve{\mathrm{s}}_{l}(\beta), \breve{\mathrm{t}}_{r}(\beta)\right]$ and $\breve{\mathrm{t}}(\beta)$ also values freely in the interval $\left[\breve{\mathrm{s}}_{l}(\beta), \breve{\mathrm{t}}_{r}(\beta)\right]$, which means the $\beta$-cuts of a IT2TrNN are $\left[\left(\left[\breve{\mathrm{s}}_{l}(\beta), \breve{\mathrm{t}}_{r}(\beta)\right]\right),\left(\left[\breve{\mathrm{s}}_{l}(\beta), \breve{\mathrm{t}}_{r}(\beta)\right]\right)\right]$ when $\beta>\psi_{\breve{\mathrm{s}}}^{L}$ and $\breve{\mathrm{s}}(\gamma)$ values freely in the interval $\left[\breve{\mathrm{s}}_{l}(\gamma), \breve{\mathrm{t}}_{r}(\gamma)\right]$ and $\breve{\mathrm{t}}(\gamma)$ also values freely in the interval $\left[\breve{\mathrm{s}}_{l}(\gamma), \breve{\mathrm{t}}_{r}(\gamma)\right]$, which means the $\gamma$-cuts of a IT2TrNN are $\left[\left(\left[\breve{\mathrm{s}}_{l}(\gamma), \breve{\mathrm{t}}_{r}(\gamma)\right]\right),\left(\left[\breve{\mathrm{s}}_{l}(\gamma), \breve{\mathrm{t}}_{r}(\gamma)\right]\right)\right]$ when $\gamma>\varphi_{\breve{\mathrm{s}}}^{L}$. In order to get rid of the overlapping of left and right intervals of $\alpha, \beta$ and $\gamma$ cuts of IT2TrNN, we make a modification of the above assumption, which assumes that $\breve{s}(\alpha)$ values freely in the interval $\left[\breve{s}_{l}(\alpha), \frac{x_{2}^{L}+x_{3}^{L}}{2}\right]$ and $\breve{\mathrm{t}}(\alpha)$ values freely in the interval $\left[\frac{x_{2}^{L}+x_{3}^{L}}{2}, \breve{\mathrm{t}}_{r}(\alpha)\right]$, when $\alpha>\phi_{\breve{\mathrm{S}}}^{L}$. Similarly, $\breve{\mathrm{s}}(\beta)$ values freely in the interval $\left[\breve{\mathrm{s}}_{l}(\beta), \frac{x_{2}^{L}+x_{3}^{L}}{2}\right]$ and $\breve{\mathrm{t}}(\beta)$ values freely in the interval $\left[\frac{x_{2}^{L}+x_{3}^{L}}{2}, \breve{\mathrm{t}}_{r}(\beta)\right]$, when $\beta>\psi_{\breve{\mathrm{s}}}^{L}$ and $\breve{\mathrm{s}}(\gamma)$ values freely in the interval $\left[\breve{\mathrm{s}}_{l}(\gamma), \frac{x_{2}^{L}+x_{3}^{L}}{2}\right]$ and $\breve{\mathrm{t}}(\gamma)$ values freely in the interval $\left[\frac{x_{2}^{L}+x_{3}^{L}}{2}, \breve{\mathrm{t}}_{r}(\gamma)\right]$, when $\gamma>\varphi_{\breve{\mathrm{S}}}^{L}$. In other words, we replace $\breve{\mathrm{s}}_{r}(\alpha)$ and $\breve{\mathrm{t}}_{l}(\alpha)$ with $\frac{x_{2}^{L}+x_{3}^{L}}{2}$ when $\alpha>\phi_{\breve{\mathrm{S}}}^{L}, \breve{\mathrm{s}}_{r}(\beta)$ and $\breve{\mathrm{t}}_{l}(\beta)$ with $\frac{x_{2}^{L}+x_{3}^{L}}{2}$ when $\beta>\psi_{\breve{\mathrm{S}}}^{L}$ and $\breve{\mathrm{s}}_{r}(\gamma)$ and $\breve{\mathrm{t}}_{l}(\gamma)$ with $\frac{x_{2}^{L}+x_{3}^{L}}{2}$ when $\gamma>\varphi_{\breve{\mathrm{S}}}^{L}$. Therefore, $\breve{\mathrm{s}}_{r}(\alpha), \breve{\mathrm{t}}_{l}(\alpha), \breve{\mathrm{s}}_{r}(\beta), \breve{\mathrm{t}}_{l}(\beta), \breve{\mathrm{s}}_{r}(\gamma)$ and $\breve{\mathrm{t}}_{l}(\gamma)$ can be redefined as follows:

$$
\breve{\mathrm{s}}_{r}(\alpha) \triangleq \begin{cases}\breve{\mathrm{S}}_{r}(\alpha) & \alpha \in\left[0, \phi_{\breve{\mathrm{S}}}^{L}\right] \\ \frac{x_{2}^{L}+x_{3}^{L}}{2} & \alpha \in\left[\phi_{\breve{\mathrm{S}}}^{L}, \phi_{\breve{\mathrm{S}}}^{U}\right]\end{cases}
$$




$$
\begin{aligned}
& \breve{\mathrm{t}}_{l}(\alpha) \triangleq \begin{cases}\breve{\mathrm{t}}_{l}(\alpha) & \alpha \in\left[0, \phi_{\breve{\mathrm{S}}}^{L}\right] \\
\frac{x_{2}^{L}+x_{3}^{L}}{2} & \alpha \in\left[\phi_{\breve{\mathrm{S}}}^{L}, \phi_{\breve{\mathrm{S}}}^{U}\right]\end{cases} \\
& \breve{\mathrm{s}}_{r}(\beta) \triangleq \begin{cases}\breve{\mathrm{s}}_{r}(\beta) & \beta \in\left[0, \psi_{\breve{\mathrm{S}}}^{L}\right] \\
\frac{x_{2}^{L}+x_{3}^{L}}{2} & \beta \in\left[\psi_{\breve{\mathrm{S}}}^{L}, \psi_{\breve{\mathrm{S}}}^{U}\right]\end{cases} \\
& \breve{\mathrm{t}}_{l}(\beta) \triangleq \begin{cases}\breve{\mathrm{t}}_{l}(\beta) & \beta \in\left[0, \psi_{\breve{\mathrm{S}}}^{L}\right] \\
\frac{x_{2}^{L}+x_{3}^{L}}{2} & \beta \in\left[\psi_{\breve{\mathrm{S}}}^{L}, \psi_{\breve{\mathrm{S}}}^{U}\right]\end{cases} \\
& \breve{\mathrm{s}}_{r}(\gamma) \triangleq \begin{cases}\breve{\mathrm{s}}_{r}(\gamma) & \gamma \in\left[0, \varphi_{\breve{\mathrm{S}}}^{L}\right] \\
\frac{x_{2}^{L}+x_{3}^{L}}{2} & \gamma \in\left[\varphi_{\breve{\mathrm{S}}}^{L}, \varphi_{\breve{\mathrm{S}}}^{U}\right]\end{cases} \\
& \breve{\mathrm{t}}_{l}(\gamma) \triangleq \begin{cases}\breve{\mathrm{t}}_{l}(\gamma) & \gamma \in\left[0, \varphi_{\breve{\mathrm{S}}}^{L}\right] \\
\frac{x_{2}^{L}+x_{3}^{L}}{2} & \gamma \in\left[\varphi_{\breve{\mathrm{S}}}^{L}, \varphi_{\breve{\mathrm{S}}}^{U}\right] .\end{cases}
\end{aligned}
$$

Figure 3 indicates a new definition of the $(\alpha, \beta, \gamma)$-cuts of a IT2TrNN when $(\alpha, \beta, \gamma)>\left(\phi_{\breve{\mathrm{S}}}^{L}, \psi_{\breve{\mathrm{S}}}^{L}, \varphi_{\breve{\mathrm{S}}}^{L}\right)$ and $(\alpha, \beta, \gamma)<\left(\phi_{\breve{\mathrm{S}}}^{L}, \psi_{\breve{\mathrm{S}}}^{L}, \varphi_{\breve{\mathrm{S}}}^{L}\right)$.

\subsection{Algorithm for calculating the distance between two IT2TrNNs}

Step 1. Computing the $(\alpha, \beta, \gamma)$-cuts of the difference between two IT2TrNNs.

For IT2TrNNs $\breve{S}$ and $\breve{T}$

$$
\begin{aligned}
\breve{\mathrm{S}} & =\left(\breve{\mathrm{S}}^{U}, \breve{\mathrm{S}}^{L}\right) \\
& =\left[\left(\breve{\mathrm{s}}_{1}^{U}, \breve{\mathrm{s}}_{2}^{U}, \breve{\mathrm{s}}_{3}^{U}, \breve{\mathrm{s}}_{4}^{U} ; \phi_{\mathrm{S}}^{U}, \psi_{\breve{\mathrm{S}}}^{U}, \varphi_{\breve{\mathrm{S}}}^{U}\right),\left(\breve{\mathrm{s}}_{1}^{L}, \breve{\mathrm{s}}_{2}^{L}, \breve{\mathrm{s}}_{3}^{L}, \breve{\mathrm{s}}_{4}^{L} ; \phi_{\breve{\mathrm{S}}}^{L}, \psi_{\mathrm{S}}^{L}, \varphi_{\breve{\mathrm{S}}}^{L}\right)\right] \\
\breve{\mathrm{T}} & =\left(\breve{\mathrm{T}}^{U}, \breve{\mathrm{T}}^{L}\right) \\
& =\left[\left(\breve{\mathrm{t}}_{1}^{U}, \breve{\mathrm{t}}_{2}^{U}, \breve{\mathrm{t}}_{3}^{U}, \breve{\mathrm{t}}_{4}^{U} ; \phi_{\breve{\mathrm{T}}}^{U}, \psi_{\breve{\mathrm{T}}}^{U}, \varphi_{\breve{\mathrm{T}}}^{U}\right),\left(\breve{\mathrm{t}}_{1}^{L}, \breve{\mathrm{t}}_{2}^{L}, \breve{\mathrm{t}}_{3}^{L}, \breve{\mathrm{t}}_{4}^{L} ; \phi_{\breve{\mathrm{T}}}^{L}, \psi_{\breve{\mathrm{T}}}^{L}, \varphi_{\breve{\mathrm{T}}}^{L}\right)\right]
\end{aligned}
$$

the difference between them can be computed by using the subtraction operation denoted as $\breve{\mathrm{S}}-\breve{\mathrm{T}}$ that is also an IT2TrNN. The $(\alpha, \beta, \gamma)$-cut of the difference between $\breve{\mathrm{S}}$ and $\breve{\mathrm{T}}$ is represented in Figure 4 denoted as follows:

$$
\begin{aligned}
& (\breve{\mathrm{S}}-\breve{\mathrm{T}})_{\alpha}=\left[\left[(\breve{\mathrm{S}}-\breve{\mathrm{T}})_{\alpha 1},(\breve{\mathrm{S}}-\breve{\mathrm{T}})_{\alpha_{2}}\right]\left[(\breve{\mathrm{S}}-\breve{\mathrm{T}})_{\alpha 3},(\breve{\mathrm{S}}-\breve{\mathrm{T}})_{\alpha 4}\right]\right] \\
& (\breve{\mathrm{S}}-\breve{\mathrm{T}})_{\beta}=\left[\left[(\breve{\mathrm{S}}-\breve{\mathrm{T}})_{\beta_{1}},(\breve{\mathrm{S}}-\breve{\mathrm{T}})_{\beta_{2}}\right]\left[(\breve{\mathrm{S}}-\breve{\mathrm{T}})_{\beta_{3}},(\breve{\mathrm{S}}-\breve{\mathrm{T}})_{\beta_{4}}\right]\right] \\
& (\breve{\mathrm{S}}-\breve{\mathrm{T}})_{\gamma}=\left[\left[(\breve{\mathrm{S}}-\breve{\mathrm{T}})_{\gamma_{1}},(\breve{\mathrm{S}}-\breve{\mathrm{T}})_{\gamma_{2}}\right]\left[(\breve{\mathrm{S}}-\breve{\mathrm{T}})_{\gamma_{3}},(\breve{\mathrm{S}}-\breve{\mathrm{T}})_{\gamma_{4}}\right]\right]
\end{aligned}
$$

Now, we have

$$
(\breve{\mathrm{S}}-\breve{\mathrm{T}})_{\alpha 1}=\frac{\left(\breve{\mathrm{s}}_{2}^{U}-\breve{\mathrm{t}}_{3}^{U}-\breve{\mathrm{s}}_{1}^{U}+\breve{\mathrm{t}}_{4}^{U}\right) \cdot \alpha}{\min \left(\phi_{\breve{\mathrm{S}}}^{U}, \phi_{\breve{\mathrm{T}}}^{U}\right)}+\breve{\mathrm{s}}_{1}^{U}-\breve{\mathrm{t}}_{4}^{U} \quad \alpha \in\left[0, \min \left(\phi_{\breve{\mathrm{S}}}^{U}, \phi_{\breve{\mathrm{T}}}^{U}\right)\right]
$$




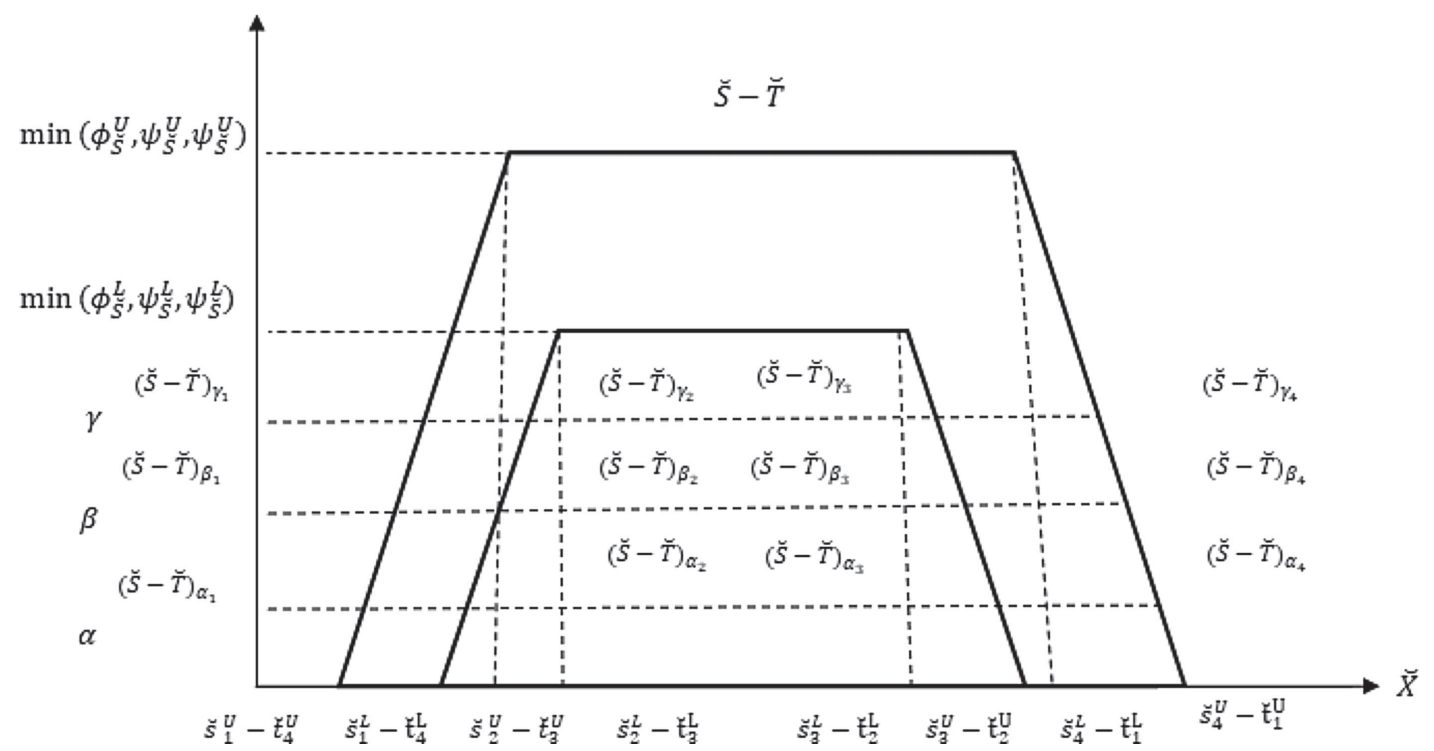

Figure 4 . The $(\alpha, \beta, \gamma)$-cut of difference between $\breve{\mathrm{S}}$ and $\breve{\mathrm{T}}$.

$$
\begin{aligned}
& (\breve{\mathrm{S}}-\breve{\mathrm{T}})_{\alpha 2}= \begin{cases}\frac{\left(\breve{\mathrm{s}}_{2}^{L}-\breve{\mathrm{t}}_{3}^{L}-\breve{\mathrm{s}}_{1}^{L}+\breve{\mathrm{t}}_{4}^{L}\right) \cdot \alpha}{\min \left(\phi_{\breve{S}}^{L}, \phi_{\breve{\mathrm{L}}}^{L}\right)}+\breve{\mathrm{s}}_{1}^{L}-\breve{\mathrm{t}}_{4}^{L} & \alpha \in\left[0, \min \left(\phi_{\breve{\mathrm{S}}}^{L}, \phi_{\breve{\mathrm{T}}}^{L}\right)\right] \\
\frac{\breve{s}_{2}^{L}-\breve{\mathrm{t}}_{3}^{L}+\breve{\mathrm{s}}_{3}^{L}-\breve{\mathrm{t}}_{2}^{L}}{2} & \alpha \in\left[\min \left(\phi_{\breve{\mathrm{S}}}^{L}, \phi_{\breve{\mathrm{T}}}^{L}\right), \min \left(\phi_{\breve{\mathrm{S}}}^{U}, \phi_{\breve{\mathrm{T}}}^{U}\right)\right]\end{cases} \\
& (\breve{\mathrm{S}}-\breve{\mathrm{T}})_{\alpha 3}= \begin{cases}\breve{\mathrm{s}}_{4}^{L}-\breve{\mathrm{t}}_{1}^{L}-\frac{\left(\breve{\mathrm{s}}_{4}^{L}-\breve{\mathrm{t}}_{1}^{L}-\breve{\mathrm{s}}_{3}^{L}+\breve{\mathrm{t}}_{2}^{L}\right) \cdot \alpha}{\min \left(\phi_{\breve{\mathrm{S}}}^{L}, \phi_{\breve{\mathrm{T}}}^{L}\right)} & \alpha \in\left[0, \min \left(\phi_{\breve{\mathrm{S}}}^{L}, \phi_{\breve{\mathrm{T}}}^{L}\right)\right] \\
\frac{\breve{\mathrm{s}}_{2}^{L}-\breve{\mathrm{t}}_{3}^{L}+\breve{\mathrm{s}}_{3}^{L}-\breve{\mathrm{t}}_{2}^{L}}{2} & \alpha \in\left[\min \left(\phi_{\breve{\mathrm{S}}}^{L}, \phi_{\breve{\mathrm{T}}}^{L}\right), \min \left(\phi_{\breve{\mathrm{S}}}^{U}, \phi_{\breve{\mathrm{T}}}^{U}\right)\right]\end{cases} \\
& (\breve{\mathrm{S}}-\breve{\mathrm{T}})_{\alpha 4}=\breve{\mathrm{s}}_{4}^{U}-\breve{\mathrm{t}}_{1}^{U}-\frac{\left(\breve{\mathrm{s}}_{4}^{U}-\breve{\mathrm{t}}_{1}^{U}-\breve{\mathrm{s}}_{3}^{U}+\breve{\mathrm{t}}_{2}^{U}\right) \cdot \alpha}{\min \left(\phi_{\breve{\mathrm{S}}}^{U}, \phi_{\breve{\mathrm{T}}}^{U}\right)} \quad \alpha \in\left[0, \min \left(\phi_{\breve{\mathrm{S}}}^{U}, \phi_{\breve{\mathrm{T}}}^{U}\right)\right]
\end{aligned}
$$

where $(\breve{\mathrm{S}}-\breve{\mathrm{T}})_{\alpha 1}<(\breve{\mathrm{S}}-\breve{\mathrm{T}})_{\alpha 2}<(\breve{\mathrm{S}}-\breve{\mathrm{T}})_{\alpha 3}<(\breve{\mathrm{S}}-\breve{\mathrm{T}})_{\alpha 4}$ when $\alpha \in\left[0, \min \left(\phi_{\breve{\mathrm{S}}}^{L}, \phi_{\breve{\mathrm{T}}}^{L}\right)\right]$ and $(\breve{\mathrm{S}}-\breve{\mathrm{T}})_{\alpha 1}<$ $(\breve{\mathrm{S}}-\breve{\mathrm{T}})_{\alpha 2}=(\breve{\mathrm{S}}-\breve{\mathrm{T}})_{\alpha 3}<(\breve{\mathrm{S}}-\breve{\mathrm{T}})_{\alpha 4}$ when $\alpha \in\left[\min \left(\phi_{\breve{\mathrm{S}}}^{L}, \phi_{\breve{\mathrm{T}}}^{L}\right), \min \left(\phi_{\breve{\mathrm{S}}}^{U}, \phi_{\breve{\mathrm{T}}}^{U}\right)\right]$.

Similarly,

$$
\begin{aligned}
& (\breve{\mathrm{S}}-\breve{\mathrm{T}})_{\beta_{1}}=\frac{\left(\breve{\mathrm{s}}_{2}^{U}-\breve{\mathrm{t}}_{3}^{U}-\breve{\mathrm{s}}_{1}^{U}+\breve{\mathrm{t}}_{4}^{U}\right) \cdot \beta-\breve{\mathrm{s}}_{2}^{U}+\breve{\mathrm{t}}_{3}^{U}+\left(\breve{\mathrm{s}}_{1}^{U}+\breve{\mathrm{t}}_{4}^{U}\right) \cdot \min \left(\psi_{\breve{\mathrm{S}}, U}^{U}, \psi_{\breve{\mathrm{T}}}^{U}\right)}{\min \left(\psi_{\breve{\mathrm{S}}}^{U}, \psi_{\breve{\mathrm{T}}}^{U}\right)-1} \beta \in\left[0, \min \left(\psi_{\breve{\mathrm{S}}}^{U}, \psi_{\breve{\mathrm{T}}}^{U}\right)\right] \\
& (\breve{\mathrm{S}}-\breve{\mathrm{T}})_{\beta_{2}}= \begin{cases}\frac{\left(\breve{\mathrm{s}}_{2}^{L}-\breve{\mathrm{t}}_{3}^{L}-\breve{\mathrm{s}}_{1}^{L}+\breve{\mathrm{t}}_{4}^{L}\right) \cdot \beta-\breve{\mathrm{s}}_{2}^{L}+\breve{\mathrm{t}}_{3}^{L}+\left(\breve{\mathrm{s}}_{1}^{L}+\breve{\mathrm{t}}_{4}^{L}\right) \cdot \min \left(\psi_{\breve{\mathrm{S}}, L}^{L}, \psi_{\breve{\mathrm{T}}}^{L}\right)}{\min \left(\psi_{\breve{\mathrm{S}},}^{L}, \psi_{\mathrm{T}}^{L}\right)-1} & \beta \in\left[0, \min \left(\psi_{\breve{\mathrm{S}}}^{L}, \psi_{\breve{\mathrm{T}}}^{L}\right)\right] \\
\frac{\breve{\mathrm{s}}_{2}^{L}-\breve{\mathrm{t}}_{3}^{L}+\breve{\mathrm{s}}_{3}^{L}-\breve{\mathrm{t}}_{2}^{L}}{2} & \beta \in\left[\min \left(\psi_{\breve{\mathrm{S}}}^{L}, \psi_{\breve{\mathrm{T}}}^{L}\right), \min \left(\psi_{\breve{\mathrm{S}}}^{U}, \psi_{\breve{\mathrm{T}}}^{U}\right)\right]\end{cases} \\
& (\breve{\mathrm{S}}-\breve{\mathrm{T}})_{\beta_{3}}= \begin{cases}\frac{\left(\breve{\mathrm{s}}_{4}^{L}-\breve{\mathrm{t}}_{1}^{L}-\breve{\mathrm{s}}_{3}^{L}+\breve{\mathrm{t}}_{2}^{L}\right) \cdot \beta+\breve{\mathrm{s}}_{3}^{U}-\breve{\mathrm{t}}_{2}^{L}-\left(\breve{\mathrm{s}}_{4}^{L}-\breve{\mathrm{t}}_{1}^{L}\right) \cdot \min \left(\psi_{\breve{\mathrm{S}}}^{L}, \psi_{\breve{\mathrm{T}}}^{L}\right)}{1-\min \left(\psi_{\breve{\mathrm{S}},}^{L}, \psi_{\breve{\mathrm{T}}}^{L}\right)} & \beta \in\left[0, \min \left(\psi_{\breve{\mathrm{S}}}^{L}, \psi_{\breve{\mathrm{T}}}^{L}\right)\right] \\
\frac{\breve{\mathrm{s}}_{2}^{L}-\breve{\mathrm{t}}_{3}^{L}+\breve{\mathrm{s}}_{3}^{L}-\breve{\mathrm{t}}_{2}^{L}}{2} & \beta \in\left[\min \left(\psi_{\breve{\mathrm{S}}}^{L}, \psi_{\breve{\mathrm{T}}}^{L}\right), \min \left(\psi_{\breve{\mathrm{S}}}^{U}, \psi_{\breve{\mathrm{T}}}^{U}\right)\right]\end{cases}
\end{aligned}
$$




$$
(\breve{\mathrm{S}}-\breve{\mathrm{T}})_{\beta_{4}}=\frac{\left(\breve{\mathrm{s}}_{4}^{U}-\breve{\mathrm{t}}_{1}^{U}-\breve{\mathrm{s}}_{3}^{U}+\breve{\mathrm{t}}_{2}^{U}\right) \cdot \beta+\breve{\mathrm{s}}_{3}^{U}-\breve{\mathrm{t}}_{2}^{U}-\left(\breve{\mathrm{s}}_{4}^{U}-\breve{\mathrm{t}}_{1}^{U}\right) \cdot \min \left(\psi_{\breve{\mathrm{S}}, U}^{U}, \psi_{\breve{\mathrm{T}}}^{U}\right)}{1-\min \left(\psi_{\breve{\mathrm{S}}}^{U}, \psi_{\breve{\mathrm{T}}}^{U}\right)} \beta \in\left[0, \min \left(\psi_{\breve{\mathrm{S}}}^{U}, \psi_{\breve{\mathrm{T}}}^{U}\right)\right]
$$

where $(\breve{\mathrm{S}}-\breve{\mathrm{T}})_{\beta_{1}}<(\breve{\mathrm{S}}-\breve{\mathrm{T}})_{\beta_{2}}<(\breve{\mathrm{S}}-\breve{\mathrm{T}})_{\beta_{3}}<(\breve{\mathrm{S}}-\breve{\mathrm{T}})_{\beta_{4}}$ when $\beta \in\left[0, \min \left(\psi_{\breve{\mathrm{S}}}^{L}, \psi_{\breve{\mathrm{T}}}^{L}\right)\right]$ and $(\breve{\mathrm{S}}-\breve{\mathrm{T}})_{\beta_{1}}<$ $(\breve{\mathrm{S}}-\breve{\mathrm{T}})_{\beta_{2}}=(\breve{\mathrm{S}}-\breve{\mathrm{T}})_{\beta_{3}}<(\breve{\mathrm{S}}-\breve{\mathrm{T}})_{\beta_{4}}$ when $\beta \in\left[\min \left(\psi_{\breve{\mathrm{S}}}^{L}, \psi_{\breve{\mathrm{T}}}^{L}\right), \min \left(\psi_{\breve{\mathrm{S}}}^{U}, \psi_{\breve{\mathrm{T}}}^{U}\right)\right]$, and

$$
\begin{aligned}
& (\breve{\mathrm{S}}-\breve{\mathrm{T}})_{\gamma_{1}}=\frac{\left(\breve{\mathrm{s}}_{2}^{U}-\breve{\mathrm{t}}_{3}^{U}-\breve{\mathrm{s}}_{1}^{U}+\breve{\mathrm{t}}_{4}^{U}\right) \cdot \gamma-\breve{\mathrm{s}}_{2}^{U}+\breve{\mathrm{t}}_{3}^{U}+\left(\breve{\mathrm{s}}_{1}^{U}+\breve{\mathrm{t}}_{4}^{U}\right) \cdot \min \left(\varphi_{\breve{\mathrm{S}}}^{U}, \varphi_{\breve{\mathrm{T}}}^{U}\right)}{\min \left(\varphi_{\breve{\mathrm{S}}}^{U}, \varphi_{\breve{\mathrm{T}}}^{U}\right)-1} \gamma \in\left[0, \min \left(\varphi_{\breve{\mathrm{S}}}^{U}, \varphi_{\breve{\mathrm{T}}}^{U}\right)\right] \\
& (\breve{\mathrm{S}}-\breve{\mathrm{T}})_{\gamma_{2}}= \begin{cases}\frac{\left(\breve{\mathrm{s}}_{2}^{L}-\breve{\mathrm{t}}_{3}^{L}-\breve{\mathrm{s}}_{1}^{L}+\breve{\mathrm{t}}_{4}^{L}\right) \cdot \gamma-\breve{\mathrm{s}}_{2}^{L}+\breve{\mathrm{t}}_{3}^{L}+\left(\breve{\mathrm{s}}_{1}^{L}+\breve{\mathrm{t}}_{4}^{L}\right) \cdot \min \left(\varphi_{\breve{\mathrm{S}}}^{L}, \varphi_{\overparen{\mathrm{T}}}^{L}\right)}{\min \left(\varphi_{\breve{\mathrm{S}}}^{L}, \varphi_{\breve{\mathrm{T}}}^{L}\right)-1} & \gamma \in\left[0, \min \left(\varphi_{\breve{\mathrm{S}}}^{L}, \varphi_{\breve{\mathrm{T}}}^{L}\right)\right] \\
\frac{\breve{\breve{\mathrm{s}}}_{2}^{L}-\breve{\mathrm{t}}_{3}^{L}+\breve{\mathrm{s}}_{3}^{L}-\breve{\mathrm{t}}_{2}^{L}}{2} & \gamma \in\left[\min \left(\varphi_{\breve{\mathrm{S}}}^{L}, \varphi_{\breve{\mathrm{T}}}^{L}\right), \min \left(\varphi_{\breve{\mathrm{S}}}^{U}, \varphi_{\breve{\mathrm{T}}}^{U}\right)\right]\end{cases} \\
& (\breve{\mathrm{S}}-\breve{\mathrm{T}})_{\gamma_{3}}= \begin{cases}\frac{\left(\breve{\mathrm{s}}_{4}^{L}-\breve{\mathrm{t}}_{1}^{L}-\breve{\mathrm{s}}_{3}^{L}+\breve{\mathrm{t}}_{2}^{L}\right) \cdot \gamma+\breve{\mathrm{s}}_{3}^{U}-\breve{\mathrm{t}}_{2}^{L}-\left(\breve{\mathrm{s}}_{4}^{L}-\breve{\mathrm{t}}_{1}^{L}\right) \cdot \min \left(\varphi_{\breve{\mathrm{S}}}^{L}, \varphi_{\overparen{\mathrm{T}}}^{L}\right)}{1-\min \left(\varphi_{\breve{\mathrm{S}}}^{L}, \varphi_{\breve{\mathrm{T}}}^{L}\right)} & \gamma \in\left[0, \min \left(\varphi_{\breve{\mathrm{S}}}^{L}, \varphi_{\breve{\mathrm{T}}}^{L}\right)\right] \\
\frac{\breve{\mathrm{s}}_{2}^{L}-\breve{\mathrm{t}}_{3}^{L}+\breve{\mathrm{s}}_{3}^{L}-\breve{\mathrm{t}}_{2}^{L}}{2} & \gamma \in\left[\min \left(\varphi_{\breve{\mathrm{S}}}^{L}, \varphi_{\breve{\mathrm{T}}}^{L}\right), \min \left(\varphi_{\breve{\mathrm{S}}}^{U}, \varphi_{\breve{\mathrm{T}}}^{U}\right)\right]\end{cases} \\
& (\breve{\mathrm{S}}-\breve{\mathrm{T}})_{\gamma_{4}}=\frac{\left(\breve{\mathrm{s}}_{4}^{U}-\breve{\mathrm{t}}_{1}^{U}-\breve{\mathrm{s}}_{3}^{U}+\breve{\mathrm{t}}_{2}^{U}\right) \cdot \gamma+\breve{\mathrm{s}}_{3}^{U}-\breve{\mathrm{t}}_{2}^{U}-\left(\breve{\mathrm{s}}_{4}^{U}-\breve{\mathrm{t}}_{1}^{U}\right) \cdot \min \left(\varphi_{\breve{\mathrm{S}}}^{U}, \varphi_{\breve{\mathrm{T}}}^{U}\right)}{1-\min \left(\varphi_{\breve{\mathrm{S}}}^{U}, \varphi_{\breve{\mathrm{T}}}^{U}\right)} \gamma \in\left[0, \min \left(\varphi_{\breve{\mathrm{S}}}^{U}, \varphi_{\breve{\mathrm{T}}}^{U}\right)\right]
\end{aligned}
$$

where $(\breve{\mathrm{S}}-\breve{\mathrm{T}})_{\gamma_{1}}<(\breve{\mathrm{S}}-\breve{\mathrm{T}})_{\gamma_{2}}<(\breve{\mathrm{S}}-\breve{\mathrm{T}})_{\gamma_{3}}<(\breve{\mathrm{S}}-\breve{\mathrm{T}})_{\gamma_{4}}$ when $\gamma \in\left[0, \min \left(\varphi_{\breve{\mathrm{S}}}^{L}, \varphi_{\breve{\mathrm{T}}}^{L}\right)\right]$ and $(\breve{\mathrm{S}}-\breve{\mathrm{T}})_{\gamma_{1}}<$ $(\breve{\mathrm{S}}-\breve{\mathrm{T}})_{\gamma_{2}}=(\breve{\mathrm{S}}-\breve{\mathrm{T}})_{\gamma_{3}}<(\breve{\mathrm{S}}-\breve{\mathrm{T}})_{\gamma_{4}}$ when $\gamma \in\left[\min \left(\varphi_{\breve{\mathrm{S}}}^{L}, \varphi_{\breve{\mathrm{T}}}^{L}\right), \min \left(\varphi_{\breve{\mathrm{S}}}^{U}, \varphi_{\breve{\mathrm{T}}}^{U}\right)\right]$.

Step 2. Calculating the distance between two IT2TrNNs at $\alpha, \beta$ and $\gamma$ level.

Further, the $(\alpha, \beta, \gamma)$-cut intervals of the difference between two IT2TrNNs are integrated within the range of $\alpha, \beta$ and $\gamma$ respectively by which the difference between two IT2TrNNs is converted into type-2 interval, calculated as follows:

$$
\begin{aligned}
\triangle_{\alpha}(\breve{\mathrm{S}}, \breve{\mathrm{T}})= & \int_{0}^{\min \left(\phi_{\breve{\mathrm{S}}}^{U}, \phi_{\breve{\mathrm{T}}}^{U}\right)}(\breve{\mathrm{S}}-\breve{\mathrm{T}})_{\alpha} \mathrm{d} \alpha \\
= & {\left[\int_{0}^{\min \left(\phi_{\breve{\mathrm{S}}}^{U}, \phi_{\breve{\mathrm{T}}}^{U}\right)}(\breve{\mathrm{S}}-\breve{\mathrm{T}})_{\alpha 1} \mathrm{~d} \alpha, \int_{0}^{\min \left(\phi_{\breve{\mathrm{S}}}^{U}, \phi_{\breve{\mathrm{T}}}^{U}\right)}(\breve{\mathrm{S}}-\breve{\mathrm{T}})_{\alpha 2} \mathrm{~d} \alpha\right] } \\
& \times\left[\int_{0}^{\min \left(\phi_{\breve{\mathrm{S}}}^{U}, \phi_{\breve{\mathrm{T}}}^{U}\right)}(\breve{\mathrm{S}}-\breve{\mathrm{T}})_{\alpha 3} \mathrm{~d} \alpha, \int_{0}^{\min \left(\phi_{\breve{\mathrm{S}}}^{U}, \phi_{\breve{\mathrm{T}}}^{U}\right)}(\breve{\mathrm{S}}-\breve{\mathrm{T}})_{\alpha 4} \mathrm{~d} \alpha\right]
\end{aligned}
$$

where

$$
\begin{aligned}
& \int_{0}^{\min \left(\phi_{\breve{\mathrm{S}}}^{U}, \phi_{\mathrm{T}}^{U}\right)}(\breve{\mathrm{S}}-\breve{\mathrm{T}})_{\alpha 1} \mathrm{~d} \alpha=\frac{\breve{\mathrm{s}}_{2}^{U}-\breve{\mathrm{t}}_{3}^{U}-\breve{\mathrm{s}}_{1}^{U}+\breve{\mathrm{t}}_{4}^{U}}{2 \cdot \min \left(\phi_{\breve{\mathrm{S}}}^{U}, \phi_{\breve{\mathrm{T}}}^{U}\right)} \\
& \int_{0}^{\min \left(\phi_{\breve{\mathrm{S}}}^{U}, \phi_{\breve{\mathrm{T}}}^{U}\right)}(\breve{\mathrm{S}}-\breve{\mathrm{T}})_{\alpha 2} \mathrm{~d} \alpha=\frac{\breve{\mathrm{s}}_{2}^{L}-\breve{\mathrm{t}}_{3}^{L}-\breve{\mathrm{s}}_{1}^{L}+\breve{\mathrm{t}}_{4}^{L}}{2 \cdot \min \left(\phi_{\breve{\mathrm{S}}}^{L}, \phi_{\breve{\mathrm{T}}}^{L}\right)}+\frac{\breve{\mathrm{s}}_{2}^{L}-\breve{\mathrm{t}}_{3}^{L}+\breve{\mathrm{s}}_{3}^{L}-\breve{\mathrm{t}}_{2}^{L}}{2 \cdot \min \left(\phi_{\breve{\mathrm{S}}}^{U}, \phi_{\breve{\mathrm{T}}}^{U}\right)}
\end{aligned}
$$




$$
\begin{aligned}
& \int_{0}^{\min \left(\phi_{\breve{\mathrm{S}}}^{U}, \phi_{\mathrm{T}}^{U}\right)}(\breve{\mathrm{S}}-\breve{\mathrm{T}})_{\alpha 3} \mathrm{~d} \alpha=\frac{\breve{\mathrm{s}}_{4}^{L}-\breve{\mathrm{t}}_{1}^{L}-\breve{\mathrm{s}}_{3}^{L}+\breve{\mathrm{t}}_{2}^{L}}{2 \cdot \min \left(\phi_{\breve{\mathrm{S}}}^{L}, \phi_{\breve{\mathrm{T}}}^{L}\right)}+\frac{\breve{\mathrm{s}}_{2}^{L}-\breve{\mathrm{t}}_{3}^{L}+\breve{\mathrm{s}}_{3}^{L}-\breve{\mathrm{t}}_{2}^{L}}{2 \cdot \min \left(\phi_{\breve{\mathrm{S}}}^{U}, \phi_{\breve{\mathrm{T}}}^{U}\right)} \\
& \int_{0}^{\min \left(\phi_{\breve{\mathrm{S}}}^{U}, \phi_{\mathrm{T}}^{U}\right)}(\breve{\mathrm{S}}-\breve{\mathrm{T}})_{\alpha 4} \mathrm{~d} \alpha=\frac{\breve{\mathrm{s}}_{4}^{U}-\breve{\mathrm{t}}_{1}^{U}+\breve{\mathrm{s}}_{3}^{U}+\breve{\mathrm{t}}_{2}^{U}}{2 \cdot \min \left(\phi_{\breve{\mathrm{S}}}^{U}, \phi_{\breve{\mathrm{T}}}^{U}\right)} .
\end{aligned}
$$

Similarly,

$$
\begin{aligned}
\triangle_{\beta}(\breve{\mathrm{S}}, \breve{\mathrm{T}})= & \int_{0}^{\min \left(\psi_{\breve{\mathrm{S}},}^{U}, \psi_{\breve{\mathrm{T}}}^{U}\right)}(\breve{\mathrm{S}}-\breve{\mathrm{T}})_{\beta} \mathrm{d} \beta \\
= & {\left[\int_{0}^{\min \left(\psi_{\breve{\mathrm{S}}}^{U}, \psi_{\mathrm{T}}^{U}\right)}(\breve{\mathrm{S}}-\breve{\mathrm{T}})_{\beta_{1}} \mathrm{~d} \beta, \int_{0}^{\min \left(\psi_{\breve{\mathrm{S}},}^{U}, \psi_{\mathrm{T}}^{U}\right)}(\breve{\mathrm{S}}-\breve{\mathrm{T}})_{\beta_{2}} \mathrm{~d} \beta\right] } \\
& \times\left[\int_{0}^{\min \left(\psi_{\breve{\mathrm{S}}}^{U}, \psi_{\mathrm{T}}^{U}\right)}(\breve{\mathrm{S}}-\breve{\mathrm{T}})_{\beta_{3}} \mathrm{~d} \beta, \int_{0}^{\min \left(\psi_{\breve{\mathrm{S}},}^{U}, \psi_{\breve{\mathrm{T}}}^{U}\right)}(\breve{\mathrm{S}}-\breve{\mathrm{T}})_{\beta_{4}} \mathrm{~d} \beta\right]
\end{aligned}
$$

where

$$
\begin{aligned}
\int_{0}^{\min \left(\psi_{\breve{\mathrm{S}}}^{U}, \psi_{\breve{\mathrm{T}}}^{U}\right)}(\breve{\mathrm{S}}-\breve{\mathrm{T}})_{\beta_{1}} \mathrm{~d} \beta=\frac{\breve{\mathrm{s}}_{2}^{U}-\breve{\mathrm{t}}_{3}^{U}-\breve{\mathrm{s}}_{1}^{U}+\breve{\mathrm{t}}_{4}^{U}}{2 \cdot\left(\min \left(\psi_{\breve{\mathrm{s}}}^{U}, \psi_{\breve{\mathrm{T}}}^{U}\right)-1\right)} \\
\int_{0}^{\min \left(\psi_{\breve{\mathrm{S}}, U}^{U}, \psi_{\breve{\mathrm{T}}}^{U}\right)}(\breve{\mathrm{S}}-\breve{\mathrm{T}})_{\beta_{2}} \mathrm{~d} \beta=\frac{\breve{\mathrm{s}}_{2}^{L}-\breve{\mathrm{t}}_{3}^{L}-\breve{\mathrm{s}}_{1}^{L}+\breve{\mathrm{t}}_{4}^{L}}{2 \cdot \min \left(\psi_{\breve{\mathrm{S}}}^{L}, \psi_{\breve{\mathrm{T}}}^{L}\right)}+\frac{\breve{\mathrm{s}}_{2}^{L}-\breve{\mathrm{t}}_{3}^{L}+\breve{\mathrm{s}}_{3}^{L}-\breve{\mathrm{t}}_{2}^{L}}{2 \cdot\left(\min \left(\psi_{\breve{\mathrm{S}}}^{U}, \psi_{\breve{\mathrm{T}}}^{U}\right)-1\right)} \\
\int_{0}^{\min \left(\psi_{\breve{\mathrm{S}}}^{U}, \psi_{\breve{\mathrm{T}}}^{U}\right)}(\breve{\mathrm{S}}-\breve{\mathrm{T}})_{\beta_{3}} \mathrm{~d} \beta=\frac{\breve{\mathrm{s}}_{4}^{L}-\breve{\mathrm{t}}_{1}^{L}-\breve{\mathrm{s}}_{3}^{L}+\breve{\mathrm{t}}_{2}^{L}}{2 \cdot\left(1-\min \left(\psi_{\breve{\mathrm{S}}}^{U}, \psi_{\breve{\mathrm{T}}}^{U}\right)\right)}+\frac{\breve{\mathrm{s}}_{2}^{L}-\breve{\mathrm{t}}_{3}^{L}+\breve{\mathrm{s}}_{3}^{L}-\breve{\mathrm{t}}_{2}^{L}}{2 \cdot \min \left(\psi_{\breve{\mathrm{S}}}^{U}, \psi_{\breve{\mathrm{T}}}^{U}\right)} \\
\int_{0}^{\min \left(\psi_{\breve{\mathrm{S}}}^{U}, \psi_{\breve{\mathrm{T}}}^{U}\right)}(\breve{\mathrm{S}}-\breve{\mathrm{T}})_{\beta_{4}} \mathrm{~d} \beta=\frac{\breve{\mathrm{s}}_{4}^{U}-\breve{\mathrm{t}}_{1}^{U}-\breve{\mathrm{s}}_{3}^{U}+\breve{\mathrm{t}}_{2}^{U}}{2 \cdot\left(1-\min \left(\psi_{\breve{\mathrm{S}}}^{U}, \psi_{\breve{\mathrm{T}}}^{U}\right)\right)}
\end{aligned}
$$

and

$$
\begin{aligned}
\triangle_{\gamma}(\breve{\mathrm{S}}, \breve{\mathrm{T}})= & \int_{0}^{\min \left(\varphi_{\breve{\mathrm{S}}}^{U}, \varphi_{\breve{\mathrm{T}}}^{U}\right)}(\breve{\mathrm{S}}-\breve{\mathrm{T}})_{\gamma} \mathrm{d} \gamma \\
= & {\left[\int_{0}^{\min \left(\varphi_{\breve{\mathrm{S}}}^{U}, \varphi_{\breve{\mathrm{T}}}^{U}\right)}(\breve{\mathrm{S}}-\breve{\mathrm{T}})_{\gamma_{1}} \mathrm{~d} \gamma, \int_{0}^{\min \left(\varphi_{\breve{\mathrm{S}}}^{U}, \varphi_{\breve{\mathrm{T}}}^{U}\right)}(\breve{\mathrm{S}}-\breve{\mathrm{T}})_{\gamma_{2}} \mathrm{~d} \gamma\right] } \\
& \times\left[\int_{0}^{\min \left(\varphi_{\breve{\mathrm{S}}}^{U}, \varphi_{\breve{\mathrm{T}}}^{U}\right)}(\breve{\mathrm{S}}-\breve{\mathrm{T}})_{\gamma_{3}} \mathrm{~d} \gamma, \int_{0}^{\min \left(\varphi_{\breve{\mathrm{S}}}^{U}, \varphi_{\breve{\mathrm{T}}}^{U}\right)}(\breve{\mathrm{S}}-\breve{\mathrm{T}})_{\gamma_{4}} \mathrm{~d} \gamma\right]
\end{aligned}
$$

where

$$
\begin{aligned}
& \int_{0}^{\min \left(\varphi_{\breve{\mathrm{S}}}^{U}, \varphi_{\overparen{\mathrm{T}}}^{U}\right)}(\breve{\mathrm{S}}-\breve{\mathrm{T}})_{\gamma_{1}} \mathrm{~d} \gamma=\frac{\breve{\mathrm{s}}_{2}^{U}-\breve{\mathrm{t}}_{3}^{U}-\breve{\mathrm{s}}_{1}^{U}+\breve{\mathrm{t}}_{4}^{U}}{2 \cdot\left(\min \left(\varphi_{\breve{\mathrm{S}}}^{U}, \varphi_{\breve{\mathrm{T}}}^{U}\right)-1\right)} \\
& \int_{0}^{\min \left(\varphi_{\breve{\mathrm{S}}}^{U}, \varphi_{\breve{\mathrm{T}}}^{U}\right)}(\breve{\mathrm{S}}-\breve{\mathrm{T}})_{\gamma_{2}} \mathrm{~d} \gamma=\frac{\breve{\mathrm{s}}_{2}^{L}-\breve{\mathrm{t}}_{3}^{L}-\breve{\mathrm{s}}_{1}^{L}+\breve{\mathrm{t}}_{4}^{L}}{2 \cdot \min \left(\varphi_{\breve{\mathrm{S}}}^{L}, \varphi_{\breve{\mathrm{T}}}^{L}\right)}+\frac{\breve{\mathrm{s}}_{2}^{L}-\breve{\mathrm{t}}_{3}^{L}+\breve{\mathrm{s}}_{3}^{L}-\breve{\mathrm{t}}_{2}^{L}}{2 \cdot\left(\min \left(\varphi_{\breve{\mathrm{S}}}^{U}, \varphi_{\breve{\mathrm{T}}}^{U}\right)-1\right)}
\end{aligned}
$$




$$
\begin{aligned}
& \int_{0}^{\min \left(\varphi_{\breve{\mathrm{S}}}^{U}, \varphi_{\mathrm{T}}^{U}\right)}(\breve{\mathrm{S}}-\breve{\mathrm{T}})_{\gamma_{3}} \mathrm{~d} \gamma=\frac{\breve{\mathrm{s}}_{4}^{L}-\breve{\mathrm{t}}_{1}^{L}-\breve{\mathrm{s}}_{3}^{L}+\breve{\mathrm{t}}_{2}^{L}}{2 \cdot\left(1-\min \left(\varphi_{\breve{\mathrm{S}}}^{U}, \varphi_{\breve{\mathrm{T}}}^{U}\right)\right)}+\frac{\breve{\mathrm{s}}_{2}^{L}-\breve{\mathrm{t}}_{3}^{L}+\breve{\mathrm{s}}_{3}^{L}-\breve{\mathrm{t}}_{2}^{L}}{2 \cdot \min \left(\varphi_{\breve{\mathrm{S}}}^{U}, \varphi_{\breve{\mathrm{T}}}^{U}\right)} \\
& \int_{0}^{\min \left(\varphi_{\breve{\mathrm{S}}}^{U}, \varphi_{\breve{\mathrm{T}}}^{U}\right)}(\breve{\mathrm{S}}-\breve{\mathrm{T}})_{\gamma_{4}} \mathrm{~d} \gamma=\frac{\breve{\mathrm{s}}_{4}^{U}-\breve{\mathrm{t}}_{1}^{U}-\breve{\mathrm{s}}_{3}^{U}+\breve{\mathrm{t}}_{2}^{U}}{2 \cdot\left(1-\min \left(\varphi_{\breve{\mathrm{S}}}^{U}, \varphi_{\breve{\mathrm{T}}}^{U}\right)\right)}
\end{aligned}
$$

As $(\breve{\mathrm{S}}-\breve{\mathrm{T}})_{\alpha 1}<(\breve{\mathrm{S}}-\breve{\mathrm{T}})_{\alpha 2}<(\breve{\mathrm{S}}-\breve{\mathrm{T}})_{\alpha 3}<(\breve{\mathrm{S}}-\breve{\mathrm{T}})_{\alpha 4}$ when $\alpha \in\left[0, \min \left(\phi_{\breve{\mathrm{S}}}^{L}, \phi_{\breve{\mathrm{T}}}^{L}\right)\right]$ and $(\breve{\mathrm{S}}-\breve{\mathrm{T}})_{\alpha 1}<$ $(\breve{\mathrm{S}}-\breve{\mathrm{T}})_{\alpha 2}=(\breve{\mathrm{S}}-\breve{\mathrm{T}})_{\alpha 3}<(\breve{\mathrm{S}}-\breve{\mathrm{T}})_{\alpha 4}$ when $\alpha \in\left[\min \left(\phi_{\breve{\mathrm{S}}}^{L}, \phi_{\breve{\mathrm{T}}}^{L}\right), \min \left(\phi_{\breve{\mathrm{S}}}^{U}, \phi_{\breve{\mathrm{T}}}^{U}\right)\right]$. It can be concluded that $\int_{0}^{\min \left(\phi_{\breve{\mathrm{S}}}^{U}, \phi_{\breve{\mathrm{T}}}^{U}\right)}(\breve{\mathrm{S}}-\breve{\mathrm{T}})_{\alpha 1} \mathrm{~d} \alpha<\int_{0}^{\min \left(\phi_{\breve{\mathrm{S}}}^{U}, \phi_{\breve{\mathrm{T}}}^{U}\right)}(\breve{\mathrm{S}}-\breve{\mathrm{T}})_{\alpha 2} \mathrm{~d} \alpha<\int_{0}^{\min \left(\phi_{\breve{\mathrm{S}}}^{U}, \phi_{\breve{\mathrm{T}}}^{U}\right)}(\breve{\mathrm{S}}-\breve{\mathrm{T}})_{\alpha 3} \mathrm{~d} \alpha<\int_{0}^{\min \left(\phi_{\breve{\mathrm{S}}}^{U}, \phi_{\breve{\mathrm{T}}}^{U}\right)}$ $(\breve{\mathrm{S}}-\breve{\mathrm{T}})_{\alpha 4} \mathrm{~d} \alpha$. Exchanging the position of $\breve{\mathrm{S}}$ and $\breve{\mathrm{T}}$ in equations (3.22), (3.27) and (3.32), we obtain that:

$$
\begin{aligned}
\triangle_{\alpha}(\breve{\mathrm{T}}, \breve{\mathrm{S}})= & \int_{0}^{\min \left(\phi_{\breve{\mathrm{S}}}^{U}, \phi_{\breve{\mathrm{T}}}^{U}\right)}(\breve{\mathrm{T}}-\breve{\mathrm{S}})_{\alpha} \mathrm{d} \alpha \\
= & {\left[-\int_{0}^{\min \left(\phi_{\breve{\mathrm{S}},}^{U}, \phi_{\mathrm{T}}^{U}\right)}(\breve{\mathrm{S}}-\breve{\mathrm{T}})_{\alpha 4} \mathrm{~d} \alpha,-\int_{0}^{\min \left(\phi_{\breve{\mathrm{S}}}^{U}, \phi_{\breve{\mathrm{T}}}^{U}\right)}(\breve{\mathrm{S}}-\breve{\mathrm{T}})_{\alpha 3} \mathrm{~d} \alpha\right] } \\
& \times\left[-\int_{0}^{\min \left(\phi_{\breve{\mathrm{S}}}^{U}, \phi_{\breve{\mathrm{T}}}^{U}\right)}(\breve{\mathrm{S}}-\breve{\mathrm{T}})_{\alpha 2} \mathrm{~d} \alpha,-\int_{0}^{\min \left(\phi_{\breve{\mathrm{S}}}^{U}, \phi_{\breve{\mathrm{T}}}^{U}\right)}(\breve{\mathrm{S}}-\breve{\mathrm{T}})_{\alpha 1} \mathrm{~d} \alpha\right]
\end{aligned}
$$

Similarly,

$$
\begin{aligned}
\triangle_{\beta}(\breve{\mathrm{T}}, \breve{\mathrm{S}})= & \int_{0}^{\min \left(\psi_{\breve{\mathrm{S}}}^{U}, \psi_{\breve{\mathrm{T}}}^{U}\right)}(\breve{\mathrm{T}}-\breve{\mathrm{S}})_{\beta} \mathrm{d} \beta \\
= & {\left[-\int_{0}^{\min \left(\psi_{\breve{\mathrm{S}},}^{U}, \psi_{\breve{\mathrm{T}}}^{U}\right)}(\breve{\mathrm{S}}-\breve{\mathrm{T}})_{\beta_{4}} \mathrm{~d} \beta,-\int_{0}^{\min \left(\psi_{\breve{\mathrm{S}},}^{U}, \psi_{\breve{\mathrm{T}}}^{U}\right)}(\breve{\mathrm{S}}-\breve{\mathrm{T}})_{\beta_{3}} \mathrm{~d} \beta\right] } \\
& \times\left[-\int_{0}^{\min \left(\psi_{\breve{\mathrm{S}}}^{U}, \psi_{\breve{\mathrm{T}}}^{U}\right)}(\breve{\mathrm{S}}-\breve{\mathrm{T}})_{\beta_{2}} \mathrm{~d} \beta,-\int_{0}^{\min \left(\psi_{\breve{\mathrm{S}}}^{U}, \psi_{\breve{\mathrm{T}}}^{U}\right)}(\breve{\mathrm{S}}-\breve{\mathrm{T}})_{\beta_{1}} \mathrm{~d} \beta\right]
\end{aligned}
$$

and

$$
\begin{aligned}
& \triangle_{\gamma}(\breve{\mathrm{T}}, \breve{\mathrm{S}})=\int_{0}^{\min \left(\varphi_{\breve{\mathrm{S}}}^{U}, \varphi_{\breve{\mathrm{T}}}^{U}\right)}(\breve{\mathrm{T}}-\breve{\mathrm{S}})_{\gamma} \mathrm{d} \gamma \\
& =\left[-\int_{0}^{\min \left(\varphi_{\breve{\mathrm{S}}}^{U}, \varphi_{\breve{\mathrm{T}}}^{U}\right)}(\breve{\mathrm{S}}-\breve{\mathrm{T}})_{\gamma_{4}} \mathrm{~d} \gamma,-\int_{0}^{\min \left(\varphi_{\breve{\mathrm{S}}}^{U}, \varphi_{\breve{\mathrm{T}}}^{U}\right)}(\breve{\mathrm{S}}-\breve{\mathrm{T}})_{\gamma_{3}} \mathrm{~d} \gamma\right] \\
& \times\left[-\int_{0}^{\min \left(\varphi_{\breve{\mathrm{S}}}^{U}, \varphi_{\breve{\mathrm{T}}}^{U}\right)}(\breve{\mathrm{S}}-\breve{\mathrm{T}})_{\gamma_{2}} \mathrm{~d} \gamma,-\int_{0}^{\min \left(\varphi_{\breve{\mathrm{S}}}^{U}, \varphi_{\overparen{\mathrm{T}}}^{U}\right)}(\breve{\mathrm{S}}-\breve{\mathrm{T}})_{\gamma_{1}} \mathrm{~d} \gamma\right] .
\end{aligned}
$$

Thus, it is shown that the difference between $\breve{\mathrm{S}}$ and $\breve{\mathrm{T}}$ at $(\alpha, \beta, \gamma)$ level has an important property i.e. $\triangle_{\alpha}(\breve{\mathrm{T}}, \breve{\mathrm{S}})=-\triangle_{\alpha}(\breve{\mathrm{S}}, \breve{\mathrm{T}}), \triangle_{\beta}(\breve{\mathrm{T}}, \breve{\mathrm{S}})=-\triangle_{\beta}(\breve{\mathrm{S}}, \breve{\mathrm{T}})$ and $\triangle_{\gamma}(\breve{\mathrm{T}}, \breve{\mathrm{S}})=-\triangle_{\gamma}(\breve{\mathrm{S}}, \breve{\mathrm{T}})$. It indicates that it doesn't satisfy commutativity. However, no matter what the computational order of both is, the absolute values of the endpoints of two intervals are equal. 
Step 3. In the following step, we introduce an OWA operator for deneutrosifying the difference between two IT2TrNNs at $\alpha, \beta$ and $\gamma$ level. The distance between them is denoted by $d(\breve{\mathrm{S}}, \breve{\mathrm{T}})$ and can be determined as:

$$
\begin{aligned}
& d(\breve{\mathrm{S}}, \breve{\mathrm{T}})=\left[\mathrm{F}_{\breve{\mathrm{w}}}\left(\triangle_{\alpha}(\breve{\mathrm{S}}, \breve{\mathrm{T}})\right)\right] \\
& =\left[\mathrm{F}_{\breve{\mathrm{w}}}\left(\int_{0}^{\min \left(\phi_{\breve{\mathrm{S}}}^{U}, \phi_{\breve{\mathrm{T}}}^{U}\right)}(\breve{\mathrm{S}}-\breve{\mathrm{T}})_{\alpha 1} \mathrm{~d} \alpha, \ldots, \int_{0}^{\min \left(\phi_{\breve{\mathrm{S}}}^{U}, \phi_{\breve{\mathrm{T}}}^{U}\right)}(\breve{\mathrm{S}}-\breve{\mathrm{T}})_{\alpha 4} \mathrm{~d} \alpha\right)\right] \\
& = \begin{cases}\mathrm{F}_{\breve{\mathrm{w}}}\left(\int_{0}^{\min \left(\phi_{\breve{\mathrm{S}}}^{U}, \phi_{\breve{\mathrm{T}}}^{U}\right)}(\breve{\mathrm{S}}-\breve{\mathrm{T}})_{\alpha 1} \mathrm{~d} \alpha, \ldots, \int_{0}^{\min \left(\phi_{\breve{\mathrm{S}}}^{U}, \phi_{\breve{\mathrm{T}}}^{U}\right)}(\breve{\mathrm{S}}-\breve{\mathrm{T}})_{\alpha 4} \mathrm{~d} \alpha\right) & \text { if } \breve{\mathrm{S}}>\breve{\mathrm{T}} \\
\mathrm{F}_{\breve{\mathrm{w}}}\left(-\int_{0}^{\min \left(\phi_{\breve{\mathrm{S}}}^{U}, \phi_{\breve{\mathrm{T}}}^{U}\right)}(\breve{\mathrm{S}}-\breve{\mathrm{T}})_{\alpha 1} \mathrm{~d} \alpha, \ldots,-\int_{0}^{\min \left(\phi_{\breve{\mathrm{S}}}^{U}, \phi_{\breve{\mathrm{T}}}^{U}\right)}(\breve{\mathrm{S}}-\breve{\mathrm{T}})_{\alpha 4} \mathrm{~d} \alpha\right) & \text { if } \breve{\mathrm{S}}<\breve{\mathrm{T}}\end{cases} \\
& =\left[\mathrm{F}_{\breve{\mathrm{w}}}\left(\triangle_{\beta}(\breve{\mathrm{S}}, \breve{\mathrm{T}})\right)\right] \\
& =\left[\mathrm{F}_{\breve{\mathrm{w}}}\left(\int_{0}^{\min \left(\psi_{\breve{\mathrm{S}}}^{U}, \psi_{\breve{\mathrm{T}}}^{U}\right)}(\breve{\mathrm{S}}-\breve{\mathrm{T}})_{\beta_{1}} \mathrm{~d} \beta, \ldots, \int_{0}^{\min \left(\psi_{\breve{\mathrm{S}}}^{U}, \psi_{\breve{\mathrm{T}}}^{U}\right)}(\breve{\mathrm{S}}-\breve{\mathrm{T}})_{\beta_{4}} \mathrm{~d} \beta\right)\right]
\end{aligned}
$$

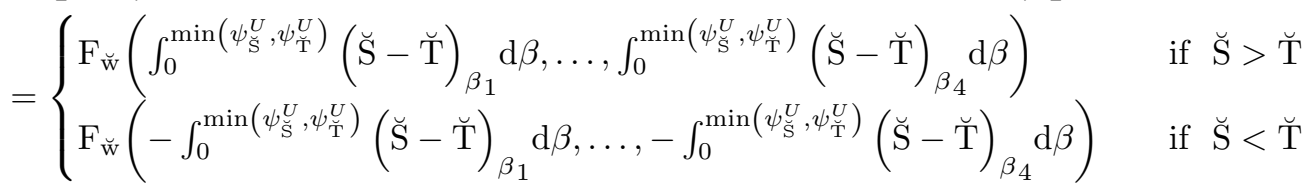

$$
\begin{aligned}
& =\left[\mathrm{F}_{\breve{\mathrm{w}}}\left(\triangle_{\gamma}(\breve{\mathrm{S}}, \breve{\mathrm{T}})\right)\right] \\
& =\left[\mathrm{F}_{\breve{\mathrm{w}}}\left(\int_{0}^{\min \left(\varphi_{\breve{\mathrm{S}}}^{U}, \varphi_{\breve{\mathrm{T}}}^{U}\right)}(\breve{\mathrm{S}}-\breve{\mathrm{T}})_{\gamma_{1}} \mathrm{~d} \gamma, \ldots, \int_{0}^{\min \left(\varphi_{\breve{\mathrm{S}}}^{U}, \varphi_{\breve{\mathrm{T}}}^{U}\right)}(\breve{\mathrm{S}}-\breve{\mathrm{T}})_{\gamma_{4}} \mathrm{~d} \gamma\right)\right] \\
& = \begin{cases}\mathrm{F}_{\breve{\mathrm{w}}}\left(\int_{0}^{\min \left(\varphi_{\breve{\mathrm{S}}}^{U}, \varphi_{\breve{\mathrm{T}}}^{U}\right)}(\breve{\mathrm{S}}-\breve{\mathrm{T}})_{\gamma_{1}} \mathrm{~d} \gamma, \ldots, \int_{0}^{\min \left(\varphi_{\breve{\mathrm{S}}}^{U}, \varphi_{\breve{\mathrm{T}}}^{U}\right)}(\breve{\mathrm{S}}-\breve{\mathrm{T}})_{\gamma_{4}} \mathrm{~d} \gamma\right) & \text { if } \breve{\mathrm{S}}>\breve{\mathrm{T}} \\
\mathrm{F}_{\breve{\mathrm{w}}}\left(-\int_{0}^{\min \left(\varphi_{\breve{\mathrm{S}}}^{U}, \varphi_{\breve{\mathrm{T}}}^{U}\right)}(\breve{\mathrm{S}}-\breve{\mathrm{T}})_{\gamma_{1}} \mathrm{~d} \gamma, \ldots,-\int_{0}^{\min \left(\varphi_{\breve{\mathrm{S}}}^{U}, \varphi_{\breve{T}}^{U}\right)}(\breve{\mathrm{S}}-\breve{\mathrm{T}})_{\gamma_{4}} \mathrm{~d} \gamma\right) & \text { if } \breve{\mathrm{S}}<\breve{\mathrm{T}}\end{cases}
\end{aligned}
$$

where $\mathrm{F}_{\breve{\mathrm{w}}}$ is an OWA operator (see Eq. (2.17)).

The degree of "orness" related with $\mathrm{F}_{\breve{\mathrm{w}}}$ is defined as:

$$
\begin{aligned}
\operatorname{orness}(\breve{\mathrm{W}}) & =\frac{1}{n-1} \sum_{i=1}^{n}(n-i) \breve{\mathrm{w}}_{i} \\
& =\breve{\mathrm{w}}_{1}+\frac{n-2}{n_{1}} \times \breve{\mathrm{w}}_{2}+\ldots+\frac{1}{n-1} \times \breve{\mathrm{w}}_{n-1}
\end{aligned}
$$

If the orness degree related to the OWA operator is greater than $\frac{1}{2}$, it depicts that the distance between two IT2TrNNs is overestimated; in contrast, if it is less than $\frac{1}{2}$, it means the distance is underestimated; further, if the orness degree is equal to $\frac{1}{2}$, it means the distance is average of the endpoints of the difference between them at $\alpha, \beta$ and $\gamma$ level.

We suppose that an OWA operator $\mathrm{F}_{\breve{\mathrm{w}}}$ is related with a weighting function $\breve{\mathrm{W}}=\left(\breve{\mathrm{w}}_{1}, \breve{\mathrm{w}}_{2}, \breve{\mathrm{w}}_{3}, \breve{\mathrm{w}}_{4}\right)$. Owing that $(\breve{\mathrm{S}}-\breve{\mathrm{T}})_{\alpha 1}<(\breve{\mathrm{S}}-\breve{\mathrm{T}})_{\alpha 2}<(\breve{\mathrm{S}}-\breve{\mathrm{T}})_{\alpha 3}<(\breve{\mathrm{S}}-\breve{\mathrm{T}})_{\alpha 4}$ and $(\breve{\mathrm{S}}-\breve{\mathrm{T}})_{\alpha 1}<(\breve{\mathrm{S}}-\breve{\mathrm{T}})_{\alpha 2}=(\breve{\mathrm{S}}-\breve{\mathrm{T}})_{\alpha 3}<(\breve{\mathrm{S}}-\breve{\mathrm{T}})_{\alpha 4}$, 
the distance between $\breve{S}$ and $\breve{T}$ can be computed as:

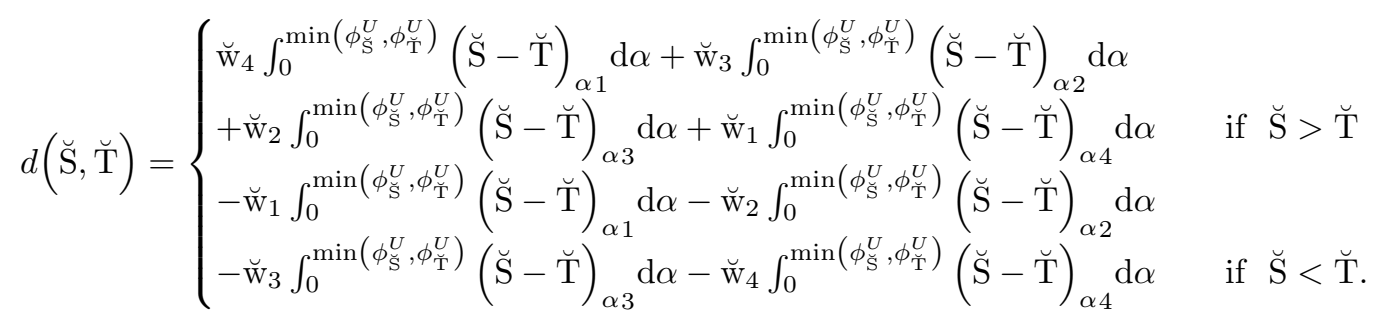

According to equations (3.10)-(3.13), we have $(\breve{\mathrm{T}}-\breve{\mathrm{S}})_{\alpha 1}=-(\breve{\mathrm{S}}-\breve{\mathrm{T}})_{\alpha 4},(\breve{\mathrm{T}}-\breve{\mathrm{S}})_{\alpha 2}=-(\breve{\mathrm{S}}-\breve{\mathrm{T}})_{\alpha 3}$, $(\breve{\mathrm{T}}-\breve{\mathrm{S}})_{\alpha 3}=-(\breve{\mathrm{S}}-\breve{\mathrm{T}})_{\alpha 2},(\breve{\mathrm{T}}-\breve{\mathrm{S}})_{\alpha 4}=-(\breve{\mathrm{S}}-\breve{\mathrm{T}})_{\alpha 1}$. Therefore, the distance between two IT2TrNNs $\breve{\mathrm{S}}$ and $\breve{\mathrm{T}}$ (Eq. (3.44)) can be computed as follows:

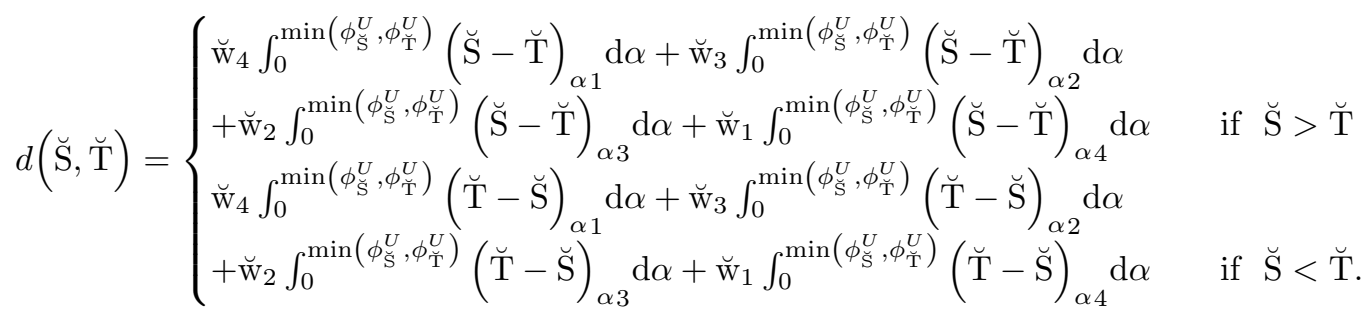

By following the same procedure as mentioned above and using equations (3.14)-(3.17) and (3.18)-(3.21). We can get

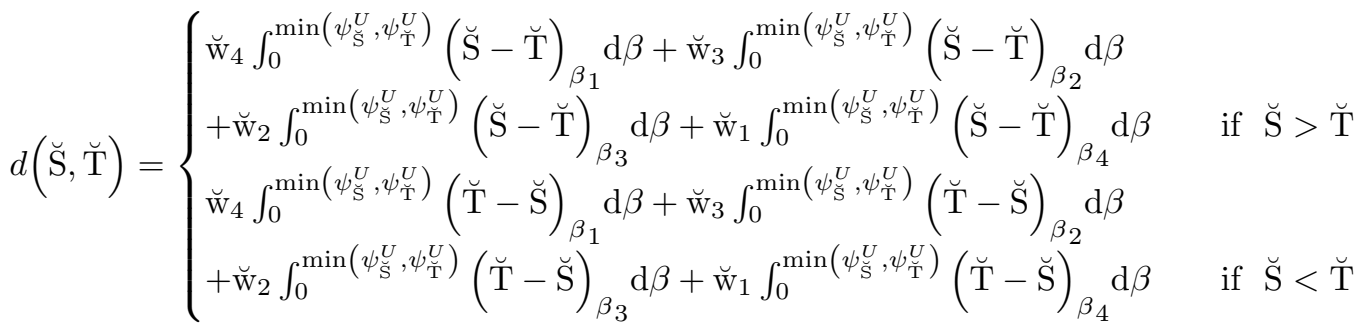

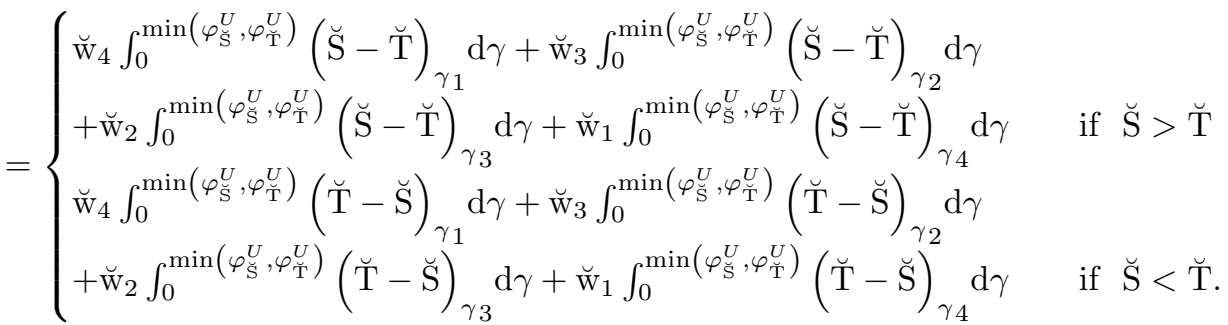

In order to acquire analytical solution of the distance between two IT2TrNNs, we suppose that $\breve{S}>\breve{T}$.

$$
\begin{aligned}
d(\breve{\mathrm{S}}, \breve{\mathrm{T}})= & w_{4} \cdot \chi_{1} \cdot \min \left(\phi_{\breve{\mathrm{S}}}^{U}, \phi_{\breve{\mathrm{T}}}^{U}\right)+w_{3} \cdot\left[\chi_{2} \cdot \min \left(\phi_{\breve{\mathrm{S}}}^{L}, \phi_{\breve{\mathrm{T}}}^{L}\right)+\sigma \cdot \min \left(\phi_{\breve{\mathrm{S}}}^{U}, \phi_{\breve{\mathrm{T}}}^{U}\right)\right] \\
& \times w_{2} \cdot\left[\chi_{3} \cdot \min \left(\phi_{\breve{\mathrm{S}}}^{L}, \phi_{\breve{\mathrm{T}}}^{L}\right)+\sigma \cdot \min \left(\phi_{\breve{\mathrm{S}}}^{U}, \phi_{\breve{\mathrm{T}}}^{U}\right)\right]+w_{1} \cdot \chi_{4} \cdot \min \left(\phi_{\breve{\mathrm{S}}}^{U}, \phi_{\breve{\mathrm{T}}}^{U}\right) \\
= & \left(w_{4} \chi_{1}+w_{3} \sigma+w_{2} \sigma+w_{1} \chi_{4}\right) \cdot \min \left(\phi_{\breve{\mathrm{S}}}^{U}, \phi_{\breve{\mathrm{T}}}^{U}\right)+\left(w_{3} \chi_{2}+w_{2} \chi_{3}\right) \cdot \min \left(\phi_{\breve{\mathrm{S}}}^{L}, \phi_{\breve{\mathrm{T}}}^{L}\right) \\
= & w_{4} \cdot \chi_{1} \cdot \min \left(\psi_{\breve{\mathrm{S}}}^{U}, \psi_{\breve{\mathrm{T}}}^{U}\right)+w_{3} \cdot\left[\chi_{2} \cdot \min \left(\psi_{\breve{\mathrm{S}}}^{L}, \psi_{\breve{\mathrm{T}}}^{L}\right)+\sigma \cdot \min \left(\psi_{\breve{\mathrm{S}}}^{U}, \psi_{\breve{\mathrm{T}}}^{U}\right)\right] \\
& \times w_{2} \cdot\left[\chi_{3} \cdot \min \left(\psi_{\breve{\mathrm{S}}}^{L}, \psi_{\breve{\mathrm{T}}}^{L}\right)+\sigma \cdot \min \left(\psi_{\breve{\mathrm{S}}}^{U}, \psi_{\breve{\mathrm{T}}}^{U}\right)\right]+w_{1} \cdot \chi_{4} \cdot \min \left(\psi_{\breve{\mathrm{S}}}^{U}, \psi_{\breve{\mathrm{T}}}^{U}\right)
\end{aligned}
$$




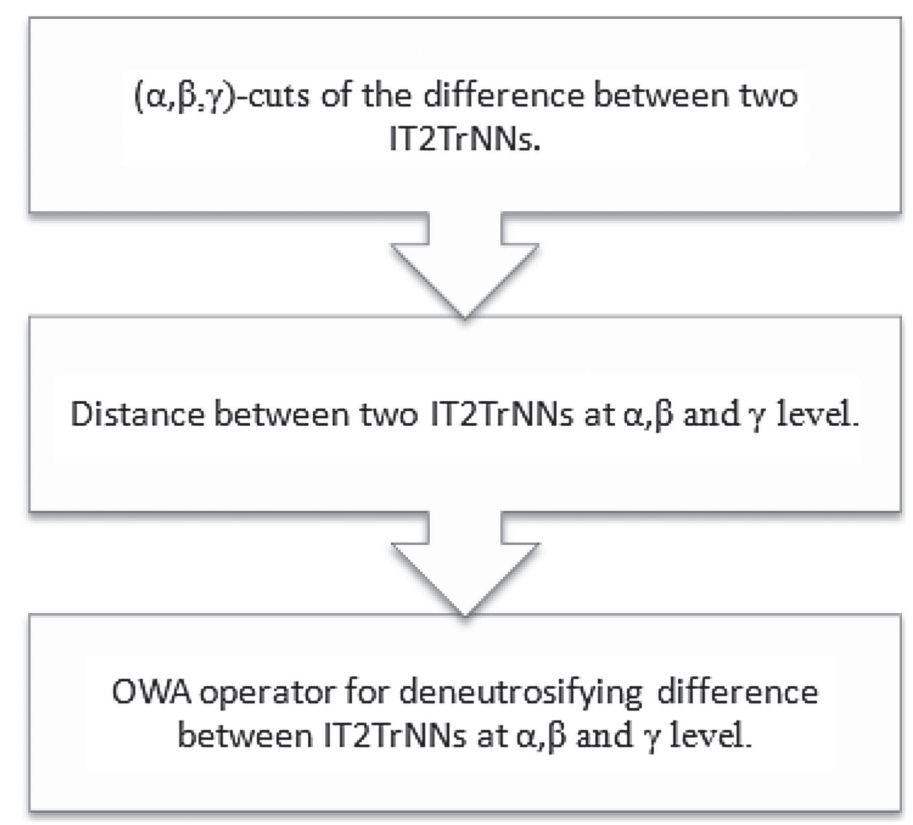

Figure 5. Flowchart for computing distance between two IT2TrNNs.

$$
\begin{aligned}
= & \left(w_{4} \chi_{1}+w_{3} \sigma+w_{2} \sigma+w_{1} \chi_{4}\right) \cdot \min \left(\psi_{\breve{\mathrm{S}}}^{U}, \psi_{\mathrm{T}}^{U}\right)+\left(w_{3} \chi_{2}+w_{2} \chi_{3}\right) \cdot \min \left(\psi_{\mathrm{S}}^{L}, \psi_{\breve{\mathrm{T}}}^{L}\right) \\
= & w_{4} \cdot \chi_{1} \cdot \min \left(\varphi_{\breve{\mathrm{S}}}^{U}, \varphi_{\breve{\mathrm{T}}}^{U}\right)+w_{3} \cdot\left[\chi_{2} \cdot \min \left(\varphi_{\breve{\mathrm{S}}}^{L}, \varphi_{\breve{\mathrm{T}}}^{L}\right)+\sigma \cdot \min \left(\varphi_{\breve{\mathrm{S}}}^{U}, \varphi_{\breve{\mathrm{T}}}^{U}\right)\right] \\
& \times w_{2} \cdot\left[\chi_{3} \cdot \min \left(\varphi_{\breve{\mathrm{S}}}^{L}, \varphi_{\breve{\mathrm{T}}}^{L}\right)+\sigma \cdot \min \left(\varphi_{\breve{\mathrm{S}}}^{U}, \varphi_{\breve{\mathrm{T}}}^{U}\right)\right]+w_{1} \cdot \chi_{4} \cdot \min \left(\varphi_{\mathrm{S}}^{U}, \varphi_{\breve{\mathrm{T}}}^{U}\right) \\
= & \left(w_{4} \chi_{1}+w_{3} \sigma+w_{2} \sigma+w_{1} \chi_{4}\right) \cdot \min \left(\varphi_{\breve{\mathrm{S}}}^{U}, \varphi_{\breve{\mathrm{T}}}^{U}\right)+\left(w_{3} \chi_{2}+w_{2} \chi_{3}\right) \cdot \min \left(\varphi_{\breve{\mathrm{S}}}^{L}, \varphi_{\breve{\mathrm{T}}}^{L}\right)
\end{aligned}
$$

where $\chi_{1}, \chi_{2}, \chi_{3}, \chi_{4}$ and $\sigma$ are constant terms calculated as follows:

$$
\begin{gathered}
\chi_{1}=\frac{\breve{\mathrm{s}}_{2}^{U}-\breve{\mathrm{t}}_{3}^{U}-\breve{\mathrm{s}}_{1}^{U}+\breve{\mathrm{t}}_{4}^{U}}{2}, \chi_{2}=\frac{\breve{\mathrm{s}}_{2}^{L}-\breve{\mathrm{t}}_{3}^{L}-\breve{\mathrm{s}}_{1}^{L}+\breve{\mathrm{t}}_{4}^{L}}{2}, \chi_{3}=\frac{\breve{\mathrm{s}}_{4}^{L}-\breve{\mathrm{t}}_{1}^{L}-\breve{\mathrm{s}}_{3}^{L}+\breve{\mathrm{t}}_{2}^{L}}{2}, \chi_{4}=\frac{\breve{\mathrm{s}}_{4}^{U}-\breve{\mathrm{t}}_{1}^{U}-\breve{\mathrm{s}}_{3}^{U}+\breve{\mathrm{t}}_{2}^{U}}{2} \\
\text { and } \sigma=\frac{\breve{\mathrm{s}}_{2}^{L}-\breve{\mathrm{t}}_{3}^{L}+\breve{\mathrm{s}}_{3}^{L}-\breve{\mathrm{t}}_{2}^{L}}{2} .
\end{gathered}
$$

$(\alpha, \beta, \gamma)$-cut and OWA operator are the core tools of this approach. Firstly, the difference between two IT2TrNNs is converted from a IT2TrNN to a type-2 interval by using the perception of $(\alpha, \beta, \gamma)$-cut. Then, the distance between two IT2TrNNs is acquired by neutrosifying the difference between them at $(\alpha, \beta, \gamma)$ level with OWA operator. By following this methodology, an analytical solution is obtained that can be implememted to TOPSIS for attaining the distances from alternatives to PIS and NIS (Fig. 5).

\section{Extension of TOPSIS With IT2TRNNs}

TOPSIS is one of the mainly used MCDM method based upon the principle of choosing an optimal alternative having shortest distance from PIS and farthest distance from NIS. In existent MCDM circumstances, decisionmakers have different decision-making outlooks towards the gains and losses: few decision-makers possess optimistic attitude, few have pessimistic while others have neutral attitude. The presented TOPSIS technique can assist decision-makers having different decision-making perspectives to make the optimal selection. Decisionmakers having optimistic outlook tend to grant more concern towards the gains than the losses so, the gains 
will be overestimated and the losses will be underestimated. The contradictory is true for the decision-makers having pessimistic outlook. Hence, decision-makers with optimistic outlook will overestimate the distance from an alternative to NIS and underestimate the distance from an alternative to the PIS. In contrast, decisionmakers holding pessimistic attitude have the opposite. In the presented approach, OWA operator is used to depict the outlook of decision-makers that can play a vital role in MCDM process. In typical TOPSIS method, the performance ratings of the local criterion related to the alternatives are articulated by crisp numbers, on the contrary, we put forward an extension of TOPSIS in the framework of IT2TrNNs to solve MCDM problems based upon the distance method introduced in Section 3.

\subsection{Algorithm of proposed TOPSIS method}

Step 1. Construct the decision matrix. Let $\left(\mathrm{L}_{1}, \ldots, \mathrm{L}_{n}\right)$ be $n$ alternatives and $\left(\mathrm{R}_{1}, \ldots, \mathrm{R}_{m}\right)$ be $m$ criterion. Let $\delta_{1}, \ldots, \delta_{m}$ be the $m$ weights associated with the criterion such that $\sum_{j=1}^{m} \delta_{j}=1$. Let $\mathrm{D}\left[\breve{\mathrm{X}}_{i j}\right]_{n \times m}$ be the decision matrix, where

$$
\begin{aligned}
& \breve{\mathrm{X}}=\left(\breve{\mathrm{X}}^{U}, \breve{\mathrm{X}}^{L}\right) \\
& =\left[\left(\breve{\mathrm{a}}_{1}^{U}, \breve{\mathrm{a}}_{2}^{U}, \breve{\mathrm{a}}_{3}^{U}, \breve{\mathrm{a}}_{4}^{U} ; \phi_{\breve{\mathrm{X}}}^{U}, \psi_{\breve{\mathrm{X}}}^{U}, \varphi_{\breve{\mathrm{X}}}^{U}\right),\left(\breve{\mathrm{a}}_{1}^{L}, \breve{\mathrm{a}}_{2}^{L}, \breve{\mathrm{a}}_{3}^{L}, \breve{\mathrm{a}}_{4}^{L} ; \phi_{\breve{\mathrm{X}}}^{L}, \psi_{\breve{\mathrm{X}}}^{L}, \varphi_{\breve{\mathrm{X}}}^{L}\right)\right] \\
& \mathrm{D}\left[\breve{\mathrm{X}}_{i j}\right]_{n \times m}=\left[\begin{array}{cccc}
{\left[\breve{\mathrm{X}}_{11}^{U}, \breve{\mathrm{X}}_{11}^{L}\right]} & {\left[\breve{\mathrm{X}}_{12}^{U}, \breve{\mathrm{X}}_{12}^{L}\right]} & \cdots & {\left[\breve{\mathrm{X}}_{1 m}^{U}, \breve{\mathrm{X}}_{1 m}^{L}\right]} \\
{\left[\breve{\mathrm{X}}_{21}^{U}, \breve{\mathrm{X}}_{21}^{L}\right]} & {\left[\breve{\mathrm{X}}_{22}^{U}, \breve{\mathrm{X}}_{22}^{L}\right]} & \cdots & {\left[\breve{\mathrm{X}}_{2 m}^{U}, \breve{\mathrm{X}}_{2 m}^{L}\right]} \\
\vdots & \vdots & \ddots & \vdots \\
{\left[\breve{\mathrm{X}}_{n 1}^{U}, \breve{\mathrm{X}}_{n 1}^{L}\right]} & {\left[\breve{\mathrm{X}}_{n 2}^{U}, \breve{\mathrm{X}}_{n 2}^{L}\right]} & \cdots & {\left[\breve{\mathrm{X}}_{n m}^{U}, \breve{\mathrm{X}}_{n m}^{L}\right]}
\end{array}\right] .
\end{aligned}
$$

Step 2. Normalize the decision matrix.

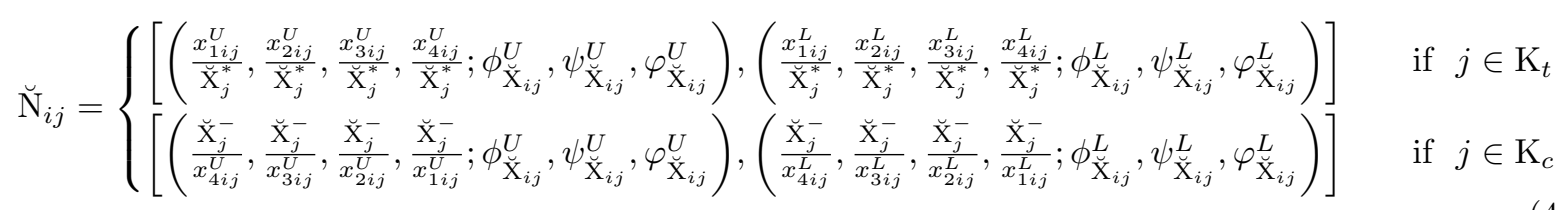

where $\breve{\mathrm{X}}_{j}^{*}=\max \breve{\mathrm{X}}_{i j}^{U}$ (for $j \in \mathrm{K}_{t}$ ) and $\breve{\mathrm{X}}_{j}^{-}=\min \breve{\mathrm{X}}_{i j}^{L}$ (for $j \in \mathrm{K}_{c}$ ), where $\mathrm{K}_{t}$ is benefit type and $\mathrm{K}_{c}$ is the cost type criterion. The normalized decision matrix is shown as follows:

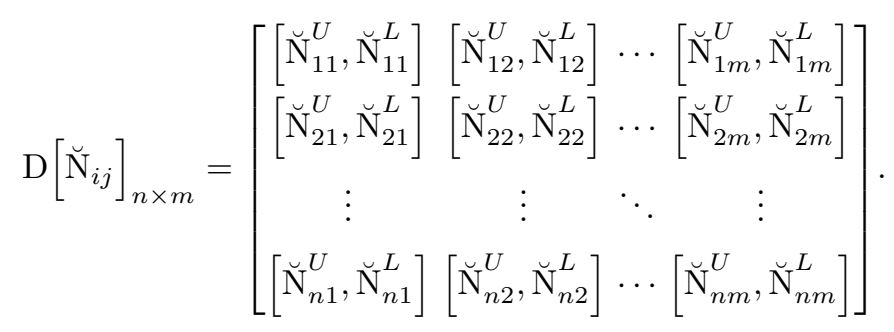

It should be known that normalization is only necessary when the criterion are estimated by using different sets of linguistic variables. Apart from that, it is not mandatory.

Step 3. Deduce the PIS and NIS.

$$
\begin{aligned}
\mathrm{P}^{*} & =\left\{v_{1}^{*}, v_{2}^{*}, \ldots, v_{m}^{*}\right\} \\
& =\left\{\left(\max _{i} \breve{\mathrm{N}}_{i j} \mid j \in \mathrm{K}_{t}\right)\left(\min _{i} \breve{\mathrm{N}}_{i j} \mid j \in \mathrm{K}_{c}\right)\right\}
\end{aligned}
$$




$$
\begin{aligned}
v_{j}^{*} & =\left[\left(v_{j 1}^{* U}, v_{j 2}^{* U}, v_{j 3}^{* U}, v_{j 4}^{* U} ; \phi_{v_{j}^{*}}^{U}, \psi_{v_{j}^{*}}^{U}, \varphi_{v_{j}^{*}}^{U}\right),\left(v_{j 1}^{* L}, v_{j 2}^{* L}, v_{j 3}^{* L}, v_{j 4}^{* L} ; \phi_{v_{j}^{*}}^{L}, \psi_{v_{j}^{*}}^{L}, \varphi_{v_{j}^{*}}^{L}\right)\right] \\
\mathrm{N}^{-} & =\left\{v_{1}^{-}, v_{2}^{-}, \ldots, v_{m}^{-}\right\} \\
& =\left\{\left(\max _{i} \breve{\mathrm{N}}_{i j} \mid j \in \mathrm{K}_{c}\right)\left(\min _{i} \breve{\mathrm{N}}_{i j} \mid j \in \mathrm{K}_{t}\right)\right\} \\
v_{j}^{-} & =\left[\left(v_{j 1}^{-U}, v_{j 2}^{-U}, v_{j 3}^{-U}, v_{j 4}^{-U} ; \phi_{v_{j}^{-}}^{U}, \psi_{v_{j}^{-}}^{U}, \varphi_{v_{j}^{-}}^{U}\right),\left(v_{j 1}^{-L}, v_{j 2}^{-L}, v_{j 3}^{-L}, v_{j 4}^{-L} ; \phi_{v_{j}^{-}}^{L}, \psi_{v_{j}^{-}}^{L}, \varphi_{v_{j}^{-}}^{L}\right)\right]
\end{aligned}
$$

where $\mathrm{K}_{t}$ is benefit type criterion set and $\mathrm{K}_{c}$ is the cost type criterion set.

Step 4. Acquire the distances among the alternatives from PIS and NIS.

According to the definitions of PIS and NIS, the performance ratings of local criterion of PIS should not be less than that of the prevailing alternatives if the criterion are benefit type however the converse is true if the criterion are cost type. Alternatively, the performance ratings of local criterion of NIS should not be more than that of the existing alternatives if the criterion are benefit type although the converse is true if the criterion are cost type. Hence, we have

$$
\begin{gathered}
v_{j}^{*} \geq \breve{\mathrm{N}}_{i j}, j \in \mathrm{K}_{t} ; \breve{\mathrm{N}}_{i j} \geq v_{j}^{*}, j \in \mathrm{K}_{c} ; \\
\breve{\mathrm{N}}_{i j} \geq v_{j}^{-}, j \in \mathrm{K}_{t} ; v_{j}^{-} \geq \breve{\mathrm{N}}_{i j}, j \in \mathrm{K}_{c} .
\end{gathered}
$$

Assuming $\mathrm{F}_{\breve{\mathrm{w}}}$ is an OWA operator having the weighting function $\breve{\mathrm{W}}=\left(\breve{\mathrm{w}}_{1}, \breve{\mathrm{w}}_{2}, \ldots, \breve{\mathrm{w}}_{n}\right)$, the distances from ratings of local criterion of the prevailing alternatives to that of PIS and NIS can be obtained by equations (3.45)-(3.47) as follows:

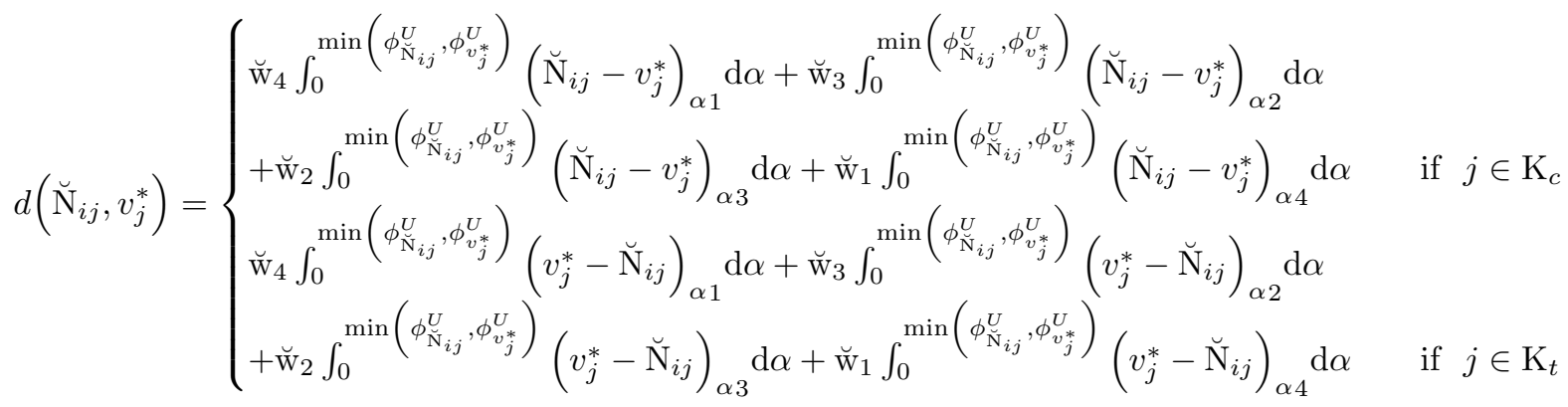

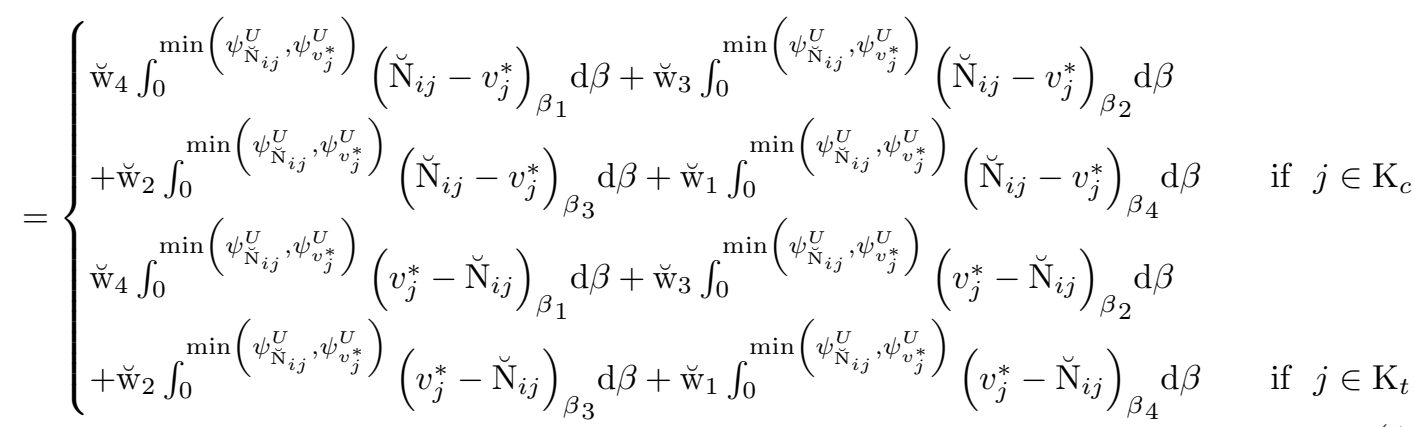




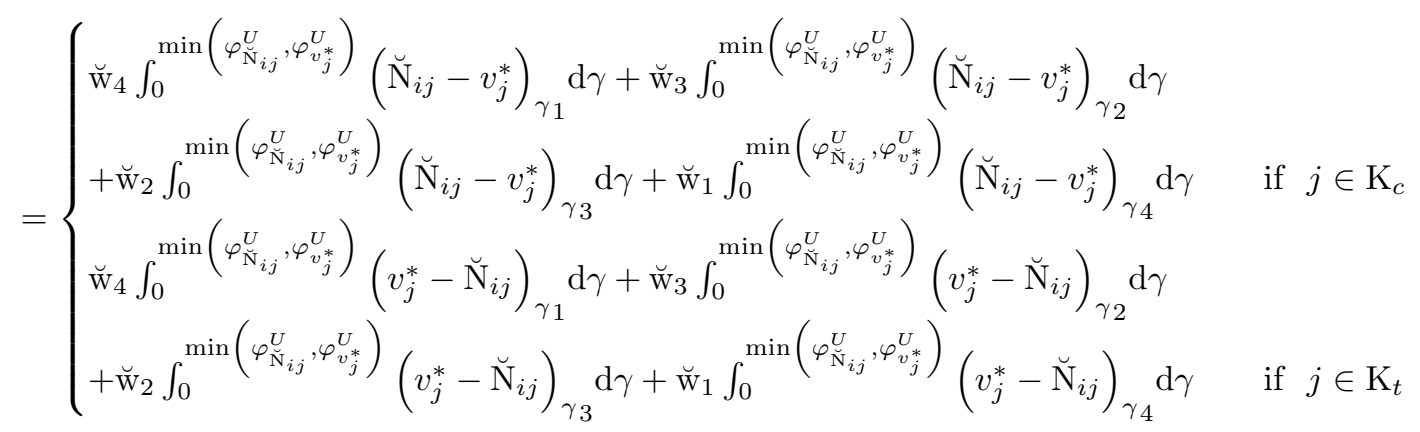

where $0<\operatorname{orness}(\breve{W})<\frac{1}{2}$ for decision-makers having optimistic attitude, $\frac{1}{2}<\operatorname{orness}(\breve{W})<1$ for decisionmakers possessing pessimistic attitude and orness $(\breve{W})=\frac{1}{2}$ for decision-makers having neutral attitude.

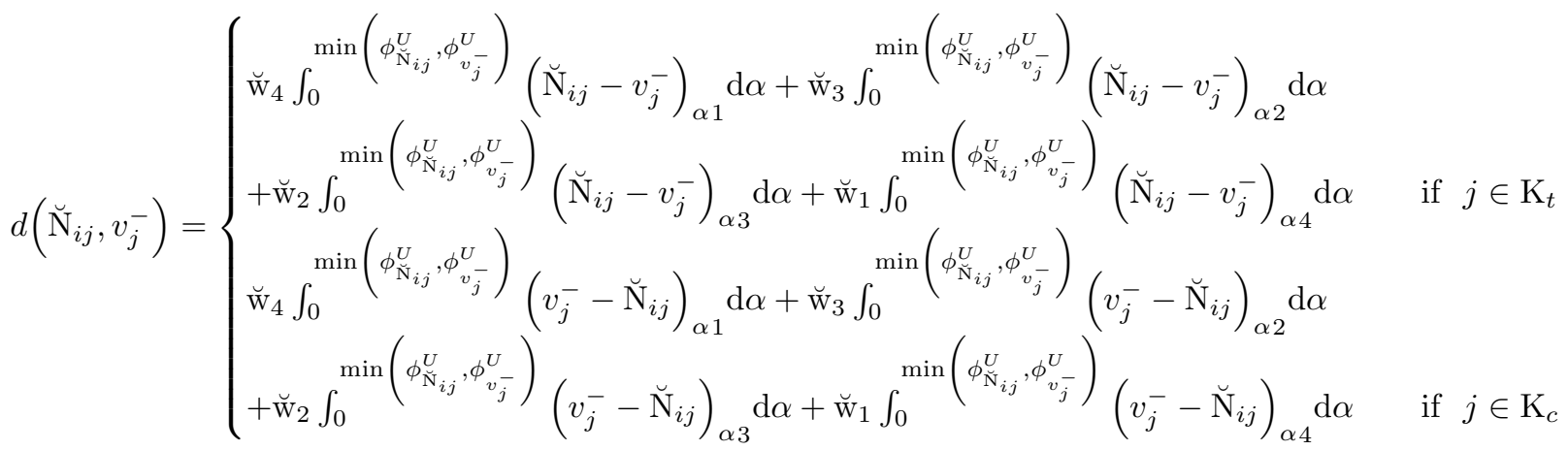

$$
\begin{aligned}
& =\left\{\begin{array}{l}
\min \left(\psi_{\mathrm{N}_{i j}}^{U}, \psi_{v_{j}^{-}}^{U}\right)\left(\breve{\mathrm{N}}_{i j}-v_{j}^{-}\right)_{\beta_{1}} \mathrm{~d} \beta+\breve{\mathrm{w}}_{3} \int_{0}^{\min \left(\psi_{\mathrm{N}_{i j}}^{U}, \psi_{v_{j}^{-}}^{U}\right)}\left(\breve{\mathrm{N}}_{i j}-v_{j}^{-}\right)_{\beta_{2}} \mathrm{~d} \beta \\
+\breve{\mathrm{w}}_{2} \int_{0}^{\min \left(\psi_{\mathrm{N}_{i j}}^{U}, \psi_{v_{j}^{-}}^{U}\right)}\left(\breve{\mathrm{N}}_{i j}-v_{j}^{-}\right)_{\beta_{3}} \mathrm{~d} \beta+\breve{\mathrm{w}}_{1} \int_{0}^{\min \left(\psi_{\mathrm{N}_{i j}}^{U}, \psi_{v_{j}^{-}}^{U}\right)}\left(\breve{\mathrm{N}}_{i j}-v_{j}^{-}\right)_{\beta_{4}} \mathrm{~d} \beta \quad \text { if } j \in \mathrm{K}_{t} \\
\min \left(\psi_{\mathrm{N}_{i j}, \psi_{v_{j}^{-}}^{U}}^{U}\right)\left(v_{j}^{-}-\breve{\mathrm{N}}_{i j}\right)_{\beta_{1}} \mathrm{~d} \beta+\breve{\mathrm{w}}_{3} \int_{0}^{\min \left(\psi_{\mathrm{N}_{i j}}^{U}, \psi_{v_{j}^{-}}^{U}\right)}\left(v_{j}^{-}-\breve{\mathrm{N}}_{i j}\right)_{\beta_{2}} \mathrm{~d} \beta \\
\breve{\mathrm{w}}_{4} \int_{0}^{\min \left(\psi_{\mathrm{N}_{i j}}^{U}, \psi_{v_{j}^{-}}^{U}\right)}\left(v_{j}^{-}-\breve{\mathrm{N}}_{i j}\right)_{\beta_{3}} \mathrm{~d} \beta+\breve{\mathrm{w}}_{1} \int_{0}^{\min \left(\psi_{\mathrm{N}_{i j}}^{U}, \psi_{v_{j}^{-}}^{U}\right)}\left(v_{j}^{-}-\breve{\mathrm{N}}_{i j}\right)_{\beta_{4}} \mathrm{~d} \beta \quad \text { if } j \in \mathrm{K}_{c} \\
+\breve{\mathrm{w}}_{2} \int_{0}
\end{array}\right.
\end{aligned}
$$




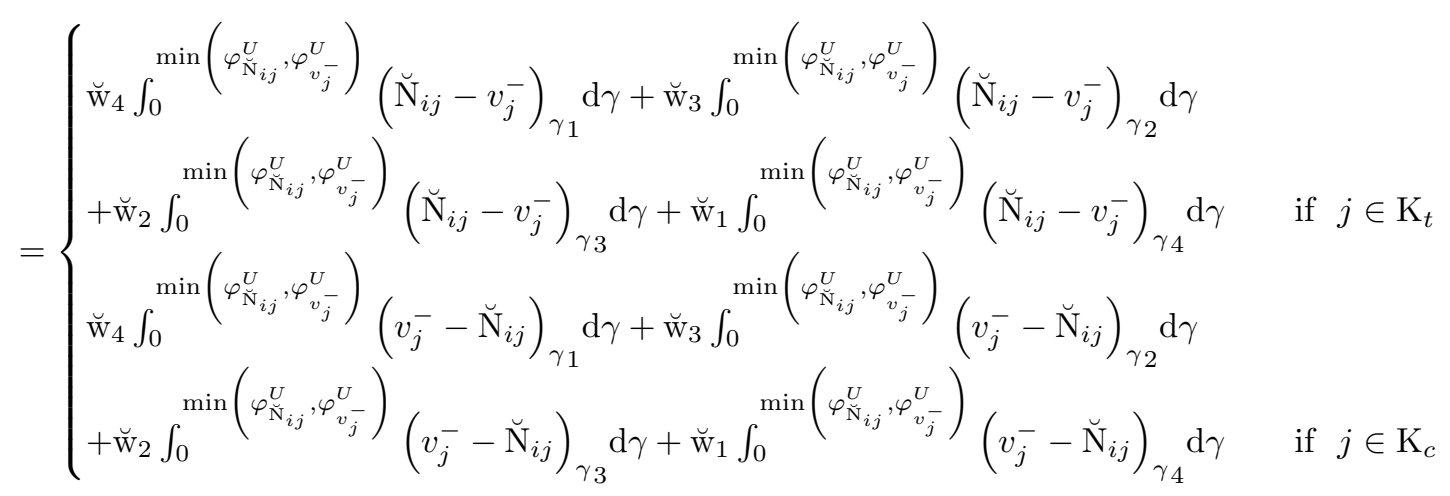

where $0<\operatorname{orness}(\breve{W})<\frac{1}{2}$ for decision-makers possessing pessimistic attitude, $\frac{1}{2}<\operatorname{orness}(\breve{W})<1$ for decision-makers holding optimistic attitude and $\operatorname{orness}(\breve{W})=\frac{1}{2}$ for decision-makers having neutral attitude. It is indicated that OWA operator depicts the perspectives of decision-makers by overestimating or underestimating the distances among the local criterion corresponding to alternatives and their ideal solutions. Further, the distances from alternatives to PIS and NIS can be computed by taking weighted sum aggregations of criterion as follows:

$$
\begin{aligned}
\mathrm{Z}_{i}^{*} & =\sum \delta_{j} d\left(v_{j}^{*}, \breve{\mathrm{N}}_{i j}\right) \\
\mathrm{Z}_{i}^{-} & =\sum \delta_{j} d\left(v_{j}^{-}, \breve{\mathrm{N}}_{i j}\right) .
\end{aligned}
$$

Step 5. Compute the relative closeness to ideal alternatives as follows:

$$
\mathrm{RC}_{i}=\frac{\mathrm{Z}_{i}^{-}}{\mathrm{Z}_{i}^{-}+\mathrm{Z}_{i}^{*}}
$$

Step 6. Rank the alternatives on the basis of their relative closeness to ideal alternatives. The alternative having the largest relative closeness coefficient is considered to be the best or optimal alternative.

\section{NUMERICAL EXAMPLE}

In the following section, we demonstrate a numerical example involving MCDM problem to achieve the practicability of our technique to the extension of TOPSIS in the framework of IT2TrNNs.

We consider an application of personnel selection in a company that is a project oriented organization and whose major focus is on project management in various fields. There are five candidates who applied for the vacant position in the company are considered as five alternatives represented as $\left(\mathrm{L}_{1}, \mathrm{~L}_{2}, \mathrm{~L}_{3}, \mathrm{~L}_{4}\right.$ and $\left.\mathrm{L}_{5}\right)$ for this MCDM problem and following are the four aspects for the evaluation of personnel by the experts panel of the company: past experience $\left(R_{1}\right)$, computer skills $\left(R_{2}\right)$, fluency in foreign language $\left(R_{3}\right)$ and project management knowledge $\left(R_{4}\right)$, where $R_{1}, R_{3}$ are cost criterion and $R_{2}, R_{4}$ are benefit criterion.

In order to pick the best candidate amongst the five personnel, first rate each of the five alternatives with regard to the four criterion and give the local criterion weights. The criterion ratings corresponding to all alternatives are provided in Table 1 and the criterion weights are shown in Table 2.

In this numerical example, ranking results are discussed in three situations: optimistic,pessimistic and neutral. Based upon our projected strategy, the methodology for choosing the best personnel is demonstrated as follows: 


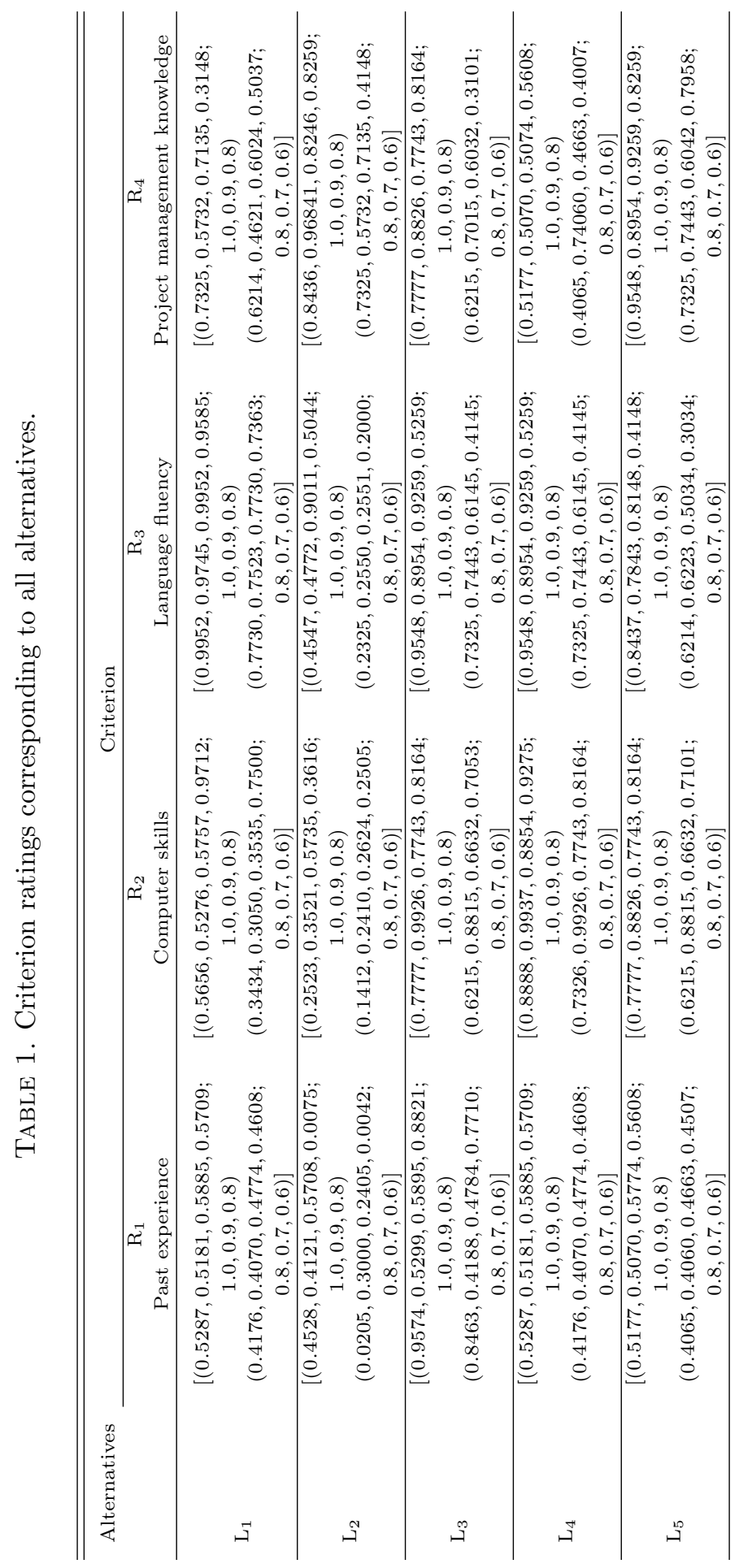


TABLE 2. Criterion weights.

\begin{tabular}{llll}
\hline \hline$\delta_{1}$ & $\delta_{2}$ & $\delta_{3}$ & $\delta_{4}$ \\
\hline 0.4 & 0.3 & 0.2 & 0.1 \\
\hline
\end{tabular}

Step 1. Build a decision matrix based on Table 1 denoted by $\mathrm{D}\left[\mathrm{X}_{i j}\right]_{5 \times 4}$ and represented as follows:

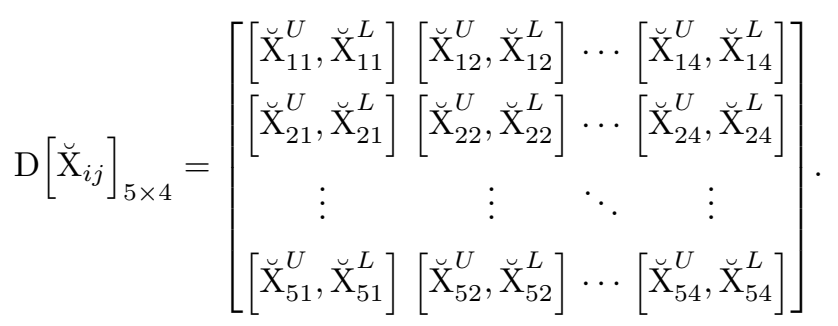

Step 2. Normalize the decision matrix.

Normalization is not necessary as the criterion in this example is assessed using the same set of linguistic variables.

Step 3. Determine the PIS and NIS respectively by using equations (4.4) and (4.5).

$$
\begin{aligned}
\mathrm{P}_{i}^{*}= & \left\{v_{1}^{*}, v_{2}^{*}, v_{3}^{*}, v_{4}^{*}\right\}=\left\{\breve{\mathrm{X}}_{21}, \breve{\mathrm{X}}_{42}, \breve{\mathrm{X}}_{23}, \breve{\mathrm{X}}_{54}\right\} \\
= & \{[(0.4528,0.4121,0.5708,0.0075 ; 1.0,0.9,0.8),(0.0205,0.3000,0.2405,0.0042 ; 0.8,0.7,0.6)], \\
& {[(0.8888,0.9937,0.8854,0.9275 ; 1.0,0.9,0.8),(0.7326,0.9926,0.7743,0.8164 ; 0.8,0.7,0.6)], } \\
& {[(0.4547,0.4772,0.9011,0.5044 ; 1.0,0.9,0.8),(0.2325,0.2550,0.2551,0.2000 ; 0.8,0.7,0.6)], } \\
& {[(0.9548,0.8954,0.9259,0.8259 ; 1.0,0.9,0.8),(0.7325,0.7443,0.6042,0.7958 ; 0.8,0.7,0.6)]\} } \\
\mathrm{N}_{i}^{-}= & \left\{v_{1}^{-}, v_{2}^{-}, v_{3}^{-}, v_{4}^{-}\right\}=\left\{\breve{\mathrm{X}}_{31}, \breve{\mathrm{X}}_{22}, \breve{\mathrm{X}}_{13}, \breve{\mathrm{X}}_{44}\right\} \\
= & {[(0.9574,0.5299,0.5895,0.8821 ; 1.0,0.9,0.8),(0.8463,0.4188,0.4784,0.7710 ; 0.8,0.7,0.6)], } \\
& {[(0.2523,0.3521,0.5735,0.3616 ; 1.0,0.9,0.8),(0.1412,0.2410,0.2624,0.2505 ; 0.8,0.7,0.6)], } \\
& {[(0.9952,0.9745,0.9952,0.9585 ; 1.0,0.9,0.8),(0.7730,0.7523,0.7730,0.7363 ; 0.8,0.7,0.6)], } \\
& {[(0.5177,0.5070,0.5074,0.5608 ; 1.0,0.9,0.8),(0.4065,0.74060,0.4663,0.4007 ; 0.8,0.7,0.6)] . }
\end{aligned}
$$

Step 4. Acquire the distances of alternatives from PIS and NIS. We allot three OWA operators with different weighting functions given as follows:

$$
\begin{aligned}
& \mathrm{F}_{\breve{\mathrm{W}}_{1}}=(0.4,0.3,0.2,0.1), \operatorname{orness}\left(\breve{\mathrm{W}}_{1}\right)=\frac{3}{4} \\
& \mathrm{~F}_{\breve{\mathrm{W}}_{2}}=(0.1,0.1,0.1,0.7), \operatorname{orness}\left(\breve{\mathrm{W}}_{2}\right)=\frac{2}{5} \\
& \mathrm{~F}_{\breve{\mathrm{W}}_{3}}=(0.1,0.2,0.3,0.4), \operatorname{orness}\left(\breve{\mathrm{W}}_{3}\right)=\frac{1}{2} .
\end{aligned}
$$

For decision-maker's optimistic attitude: the OWA operators for computing distances from ratings of local criterion to PIS and NIS are allotted with weighting functions $\breve{W}_{2}$ and $\breve{W}_{1}$ respectively. For decision-maker's pessimistic attitude: the OWA operators are appointed with weighting functions $\breve{W}_{1}$ and $\breve{W}_{2}$ respectively. For decision-maker's neutral attitude: the OWA operators are allotted with weighting functions $\breve{W}_{3}$.

Then, the distances from ratings of local criterion of all alternatives to PIS and NIS can be determined by using equations (4.6)-(4.11). Further, distances from alternatives to PIS and NIS can be acquired by using 
TABLE 3. Distances from alternatives to PIS and NIS.

\begin{tabular}{lllllllllll}
\hline \hline Alternatives & $\mathrm{L}_{1}$ & & $\mathrm{~L}_{2}$ & & $\mathrm{~L}_{3}$ & & $\mathrm{~L}_{4}$ & & $\mathrm{~L}_{5}$ & \\
\hline Distances & $\mathrm{Z}_{1}^{*}$ & $\mathrm{Z}_{1}^{-}$ & $\mathrm{Z}_{2}^{*}$ & $\mathrm{Z}_{2}^{-}$ & $\mathrm{Z}_{3}^{*}$ & $\mathrm{Z}_{3}^{-}$ & $\mathrm{Z}_{4}^{*}$ & $\mathrm{Z}_{4}^{-}$ & $\mathrm{Z}_{5}^{*}$ & $\mathrm{Z}_{5}^{-}$ \\
\hline Optimistic & 0.3457 & 0.3660 & 0.3304 & 0.3666 & 0.3346 & 0.3135 & 0.3375 & 0.3880 & 0.2908 & 0.4712 \\
Pessimistic & 0.3968 & 0.3584 & 0.4058 & 0.3986 & 0.2729 & 0.2797 & 0.2470 & 0.4104 & 0.2933 & 0.4427 \\
Neutral & 0.3776 & 0.4938 & 0.3706 & 0.3492 & 0.2630 & 0.3174 & 0.3194 & 0.4139 & 0.3102 & 0.4667 \\
\hline
\end{tabular}

TABLE 4. Relative closeness coefficients of all alternatives.

\begin{tabular}{llllll}
\hline & $\mathrm{RC}_{1}$ & $\mathrm{RC}_{2}$ & $\mathrm{RC}_{3}$ & $\mathrm{RC}_{4}$ & $\mathrm{RC}_{5}$ \\
\hline Optimistic & 0.5142 & 0.5259 & 0.4837 & 0.5348 & 0.6183 \\
Pessimistic & 0.4745 & 0.4955 & 0.5061 & 0.6242 & 0.6014 \\
Neutral & 0.5666 & 0.4851 & 0.5468 & 0.5644 & 0.6007 \\
\hline
\end{tabular}

equations (4.12) and (4.13) and the distances in three situations of decision-maker's attitude are presented in Table 3.

Step 5. Compute the relative closeness of alternatives by using equation (4.14) and the outcomes are given in Table 4.

Step 6. Rank the alternatives. They are ranked according to the descending order of their relative closeness coefficients.

Notice that the ranking results of five personnel varies for different decision-makers having different decisionmaking perspective. Decision-maker possessing optimistic attitude have following priorities for the personnel choice: $\mathrm{L}_{5}, \mathrm{~L}_{4}, \mathrm{~L}_{2}, \mathrm{~L}_{1}$, and $\mathrm{L}_{3}$. Decision-maker with pessimistic attitude holds following priorities: $\mathrm{L}_{4}, \mathrm{~L}_{5}, \mathrm{~L}_{3}, \mathrm{~L}_{2}$ and $\mathrm{L}_{1}$. Decision-maker with neutral attitude: $\mathrm{L}_{5}, \mathrm{~L}_{1}, \mathrm{~L}_{4}, \mathrm{~L}_{3}$ and $\mathrm{L}_{2}$.

In the real decision-making situation, decision-makers are not usually objective i.e. their decision-making perspective is not neutral. Hence, in such cases where their attitude is optimistic or pessimistic have more realistic importance.

\section{Comparative ANALYSiS}

A comparative revision is conducted to verify the consequences of the projected technique with several other approaches. This investigation is based on the same input information presented in Section 5.

We have chosen two existing distance approaches from literature: a signed-distance based approach for TOPSIS and a distance operational rule described as follows:

\section{- Signed distances approach:}

The signed distance of a IT2TrNN $\breve{S}$ far from $\breve{0}$ is defined as:

$$
\begin{aligned}
d\left(\breve{\mathrm{S}}, \breve{0}_{1}\right)= & \frac{1}{8}\left[2\left(\breve{\mathrm{s}}_{1}^{U}+\breve{\mathrm{s}}_{2}^{U}+\breve{\mathrm{s}}_{3}^{U}+\breve{\mathrm{s}}_{4}^{U}\right)+2\left(\breve{\mathrm{s}}_{1}^{L}+\breve{\mathrm{s}}_{2}^{L}+\breve{\mathrm{s}}_{3}^{L}+\breve{\mathrm{s}}_{4}^{L}\right)\right. \\
& \left.+3\left(\breve{\mathrm{s}}_{2}^{L}+\breve{\mathrm{s}}_{3}^{L}-\breve{\mathrm{s}}_{1}^{L}-\breve{\mathrm{s}}_{4}^{L}\right)\left(1+\frac{\phi_{\breve{\mathrm{S}}}^{L}}{\phi_{\breve{\mathrm{S}}}^{U}}-\frac{\psi_{\breve{\mathrm{S}}}^{L}}{\psi_{\breve{\mathrm{S}}}^{U}}-\frac{\varphi_{\breve{\mathrm{S}}}^{L}}{\varphi_{\bar{N}}^{U}}\right)\right] .
\end{aligned}
$$

Taking into account the above signed distance, the distance between two IT2TrNNs $\breve{\mathrm{S}}$ and $\breve{\mathrm{T}}$ can be determined as:

$$
d(\breve{\mathrm{S}}, \breve{\mathrm{T}})=\left|d\left(\breve{\mathrm{S}}, \breve{0}_{1}\right)-d\left(\breve{\mathrm{T}}, \breve{0}_{1}\right)\right| \text {. }
$$


TABLE 5. Relative closeness coefficients using signed distance-based method.

\begin{tabular}{lllll}
\hline \hline $\mathrm{RC}_{1}$ & $\mathrm{RC}_{2}$ & $\mathrm{RC}_{3}$ & $\mathrm{RC}_{4}$ & $\mathrm{RC}_{5}$ \\
\hline 0.7484 & 0.7151 & 0.8277 & 0.9501 & 0.8657 \\
\hline
\end{tabular}

TABLE 6. Relative closeness coefficients using distance operational rule.

\begin{tabular}{lllll}
\hline \hline $\mathrm{RC}_{1}$ & $\mathrm{RC}_{2}$ & $\mathrm{RC}_{3}$ & $\mathrm{RC}_{4}$ & $\mathrm{RC}_{5}$ \\
\hline 0.4234 & 0.5616 & 0.5120 & 0.4629 & 0.4825 \\
\hline
\end{tabular}

TABLE 7. Comparison of relative closeness coefficients of all alternatives.

\begin{tabular}{lccccc}
\hline \hline & $\mathrm{RC}_{1}$ & $\mathrm{RC}_{2}$ & $\mathrm{RC}_{3}$ & $\mathrm{RC}_{4}$ & $\mathrm{RC}_{5}$ \\
\hline Proposed method & & & & & \\
Optimistic & 0.5142 & 0.5259 & 0.4837 & 0.5348 & 0.6183 \\
Pessimistic & 0.4745 & 0.4955 & 0.5061 & 0.6242 & 0.6014 \\
Neutral & 0.5666 & 0.4851 & 0.5468 & 0.5644 & 0.6007 \\
\hline Signed distance-based method & 0.7484 & 0.7151 & 0.8277 & 0.9501 & 0.8657 \\
\hline Distance operational rule & 0.4234 & 0.5616 & 0.5120 & 0.4629 & 0.4825 \\
\hline
\end{tabular}

\section{- Distance operational rule:}

The distance between two IT2TrNNs can be computed as:

$$
\begin{aligned}
d(\breve{\mathrm{S}}, \breve{\mathrm{T}})= & \frac{1}{8}\left(\left|\min \left(\phi_{\breve{\mathrm{S}}}^{U}, \psi_{\breve{\mathrm{S}}}^{U}, \varphi_{\breve{\mathrm{S}}}^{U}\right) \times s_{1}^{U}-\min \left(\phi_{\breve{\mathrm{T}}}^{U}, \psi_{\breve{\mathrm{T}}}^{U}, \varphi_{\breve{\mathrm{T}}}^{U}\right) \times t_{1}^{U}\right|+\mid \min \left(\phi_{\breve{\mathrm{S}}}^{U}, \psi_{\breve{\mathrm{S}}}^{U}, \varphi_{\breve{\mathrm{S}}}^{U}\right) \times s_{2}^{U}\right. \\
& -\min \left(\phi_{\breve{\mathrm{T}}}^{U}, \psi_{\breve{\mathrm{T}}}^{U}, \varphi_{\breve{\mathrm{T}}}^{U}\right) \times t_{2}^{U}|+| \min \left(\phi_{\breve{\mathrm{S}}}^{U}, \psi_{\breve{\mathrm{S}}}^{U}, \varphi_{\breve{\mathrm{S}}}^{U}\right) \times s_{3}^{U}-\min \left(\phi_{\breve{\mathrm{T}}}^{U}, \psi_{\breve{\mathrm{T}}}^{U}, \varphi_{\breve{\mathrm{T}}}^{U}\right) \times t_{3}^{U} \mid \\
& +\left|\min \left(\phi_{\breve{\mathrm{S}}}^{U}, \psi_{\breve{\mathrm{S}}}^{U}, \varphi_{\breve{\mathrm{S}}}^{U}\right) \times s_{4}^{U}-\min \left(\phi_{\breve{\mathrm{T}}}^{U}, \psi_{\breve{\mathrm{T}}}^{U}, \varphi_{\breve{\mathrm{T}}}^{U}\right) \times t_{4}^{U}\right| \\
& +\left|\min \left(\phi_{\breve{\mathrm{S}}}^{L}, \psi_{\breve{\mathrm{S}}}^{L}, \varphi_{\breve{\mathrm{S}}}^{L}\right) \times s_{1}^{L}-\min \left(\phi_{\breve{\mathrm{T}}}^{L}, \psi_{\breve{\mathrm{T}}}^{L}, \varphi_{\breve{\mathrm{T}}}^{L}\right) \times t_{1}^{L}\right| \\
& +\left|\min \left(\phi_{\breve{\mathrm{S}}}^{L}, \psi_{\breve{\mathrm{S}}}^{L}, \varphi_{\breve{\mathrm{S}}}^{L}\right) \times s_{2}^{L}-\min \left(\phi_{\breve{\mathrm{T}}}^{L}, \psi_{\breve{\mathrm{T}}}^{L}, \varphi_{\breve{\mathrm{T}}}^{L}\right) \times t_{2}^{L}\right| \\
& +\left|\min \left(\phi_{\breve{\mathrm{S}}}^{L}, \psi_{\breve{\mathrm{S}}}^{L}, \varphi_{\breve{\mathrm{S}}}^{L}\right) \times s_{3}^{L}-\min \left(\phi_{\breve{\mathrm{T}}}^{L}, \psi_{\breve{\mathrm{T}}}^{L}, \varphi_{\breve{\mathrm{T}}}^{L}\right) \times t_{3}^{L}\right| \\
& \left.+\left|\min \left(\phi_{\breve{\mathrm{S}}}^{L}, \psi_{\breve{\mathrm{S}}}^{L}, \varphi_{\breve{\mathrm{S}}}^{L}\right) \times s_{4}^{L}-\min \left(\phi_{\breve{\mathrm{T}}}^{L}, \psi_{\breve{\mathrm{T}}}^{L}, \varphi_{\breve{\mathrm{T}}}^{L}\right) \times t_{4}^{L}\right|\right) .
\end{aligned}
$$

The relative closeness coefficients computed by using the signed distance-based approach are given in Table 5 while those computed by using the distance operational rule are given in Table 6 and the comparison of the relative closeness coefficients computed by our proposed approach and other two methods are given in Table 7 .

According to the comparative study, the results show that the projected approach is way better than the other two techniques since it can produce several subjective ranking results in different situations where the decision-makers have different decision-making outlook while in both of the other approaches, attitude of the decision-makers is not considered that means the two techniques can only yield objective ranking results for the decision-makers. Though, in the existent world, the outlook of decision-maker plays a vital role while choosing the best alternative. Therefore, it is presented that our anticipated technique can produce multiple subjective decisions for the decision-makers whilst choosing the best alternative according to their decision-making 
attitude. Consequently, our presented approach is more suitable and accurate in dealing MCDM problems as it takes the outlook of decision-makers into deliberation.

\section{Conclusion}

In this article, we have developed a TOPSIS technique in the framework of IT2TrNNs. In contrast with the prevailing approaches, the novelty of this research work is to project a new distance computation strategy for IT2TrNNs using the concept of OWA operator and $(\alpha, \beta, \gamma)$-cut by which a more flexible and precise analytical solution of the distance between two IT2TrNNs is presented. Furthermore, we developed a unique TOPSIS technique for coping up with MCDM problems formed on the distance method which can produce multiple subjective decisions for the decision-makers having different decision-making perspectives. Nonetheless, it is also indicated that the weighting functions associated with the OWA operators are allocated without any examination or practice in this study that may manipulate the quality of decisions. In future, we aim to broaden our research in the other directions and its application in some other neutrosophic systems.

\section{REFERENCES}

[1] M. Akram and A. Adeel, TOPSIS approach for MAGDM based on interval-valued hesitant fuzzy N-soft environment. Int. J. Fuzzy Syst. 21 (2019) 993-1009.

[2] K.T. Atanassov, Intuitionistic fuzzy sets. Fuzzy Set. Syst. 20 (1986) 87-96.

[3] K.T. Atanassov, More on intuitionistic fuzzy sets. Fuzzy Set. Syst. 33 (1989) 37-46.

[4] K.T. Atanassov and G. Gargov, Interval valued intuitionistic fuzzy sets. Fuzzy Set. Syst. 31 (1989) $343-349$.

[5] P. Biswas, S. Pramanik and B.C. Giri, Value and ambiguity index based ranking method of single-valued trapezoidal neutrosophic numbers and its application to multi-attribute decision making. Neutrosophic Sets Syst. 12 (2016) $127-138$.

[6] P. Biswas, S. Pramanik and B.C. Giri, Distance measure based MADM strategy with interval trapezoidal neutrosophic numbers. Neutrosophic Sets Syst. 19 (2018) 40-46.

[7] P. Biswas, S. Pramanik and B.C. Giri, TOPSIS strategy for multi-attribute decision making with trapezoidal numbers. Neutrosophic Sets Syst. 19 (2018) 29-39.

[8] E. Celik, M. Gul, N. Aydin and A.T. Gumus, A comprehensive review of multi-criteria decision making approaches based on interval type-2 fuzzy sets. Knowl. Based Syst. 85 (2015) 329-341.

[9] T.Y. Chen, Signed distanced-based TOPSIS method for multiple criteria decision analysis based on generalized interval-valued fuzzy numbers. Int. J. Inf. Tech. Decis. 10 (2011) 1131-1159.

[10] T.Y. Chen, Multiple criteria group decision-making with generalized interval-valued fuzzy numbers based on signed distances and incomplete weights. Appl. Math. Model. 36 (2012) 3029-3052.

[11] T.Y. Chen, A signed distance-based approach to importance assessment and multi-criteria group decision analysis based on interval type-2 fuzzy set. Knowl. Inf. Syst. 35 (2013) 193-231.

[12] S.M. Chen, M.W. Yang, L.W. Lee and S.W. Yang, Fuzzy multiple attributes group decision-making based on ranking interval type-2 fuzzy sets. Expert Syst. Appl. 39 (2012) 5295-5308.

[13] S. Dan, M.B. Kar, S. Majumder, B. Roy, S. Kar and D. Pamucar, Intuitionistic type-2 fuzzy set. Symmetry 11 (2019) 01-18.

[14] I. Deli, Interval-valued neutrosophic soft sets and its decision making. Int. J. Mach. Learn. Cybern. 8 (2017) 665-676.

[15] I. Deli, Operators on single valued trapezoidal neutrosophic numbers and SVTN-group decision making. Neutrosophic Sets Syst. 22 (2018) 131-151.

[16] I. Deli, Some operators with IVGSVTrN-numbers and their applications to multiple criteria group decision making. Neutrosophic Sets Syst. 25 (2019) 33-53.

[17] I. Deli and Y. Subas, A ranking method of single valued neutrosophic numbers and its applications to multi-attribute decision making problems. Int. J. Mach. Learn. Cybern. 8 (2017) 1309-1322.

[18] D. Dubois and H. Prade, Fuzzy sets and systems: theory and applications. Math. Sci. Eng. 144 (1980) 01-389.

[19] H. Garg and S. Singh, A novel triangular interval type-2 intuitionistic fuzzy sets and their aggregation operators. Iran. J. Fuzzy Syst. 15 (2018) 69-93.

[20] B.C. Giri, M.U. Molla and P. Biswas, TOPSIS method for MADM based on interval trapezoidal neutrosophic numbers. Neutrosophic Sets Syst. 22 (2018) 151-167.

[21] P. Gupta, M.K. Mehlawat and N. Grover, A generalized TOPSIS method for intuitionistic fuzzy multiple attribute group decision making considering different scenarios of attributes weight information. Int. J. Fuzzy Syst. 21 (2019) $369-387$.

[22] C. Kahraman, S.C. Onar and B. Oztaysi, Fuzzy multicriteria decision-making: a literature review. Int. J. Comput. Intell. Syst. 8 (2015) 637-666.

[23] P. Liu, S. Cheng and Y. Zhang, An extended multi-criteria group decision-making PROMETHEE method based on probability multi-valued neutrosophic sets. Int. J. Fuzzy Syst. 21 (2019) 388-406. 
[24] R.X. Liang, J.Q. Wang and H.Y. Zhang, A multi-criteria decision making method based on single valued trapezoidal neutrosophic preference relation with complete weight information. Neural Comput. Appl. 30 (2018) 3383-3398.

[25] G.S. Mahapatra and T.K. Roy, Intuitionistic fuzzy number and its arithmetic operation with application on system failure. J. Uncertain Syst. 7 (2013) 92-107.

[26] J.M. Mendel, Advances in type-2 fuzzy sets and systems. Inf. Sci. 177 (2007) 84-110.

[27] J.M. Mendel and R.I. John, Type-2 fuzzy sets made simple. IEEE T. Fuzzy Syst. 10 (2002) 117-127.

[28] J.M. Mendel, R.I. John and F.L. Liu, Interval type-2 fuzzy logical systems made simple. IEEE T. Fuzzy Syst. 14 (2006) 808-821.

[29] J.H. Park, H.J. Cho and Y.C. Kwun, Extension of the VIKOR method for group decision making with interval-valued intuitionistic fuzzy information. Fuzzy Optim. Decis. Making 10 (2011) 233-253.

[30] J.J. Peng, J.Q. Wang and X.H. Chen, An extension of ELECTRE to multi-criteria decision-making problems with multihesitant fuzzy sets. Inf. Sci. 307 (2015) 113-126.

[31] S. Pramanik and R. Mallick, VIKOR based MAGDM strategy with trapezoidal neutrosophic number. Neutrosophic Sets Syst. 22 (2018) 118-130.

[32] S. Pramanik and R. Mallick, TODIM strategy for MAGDM in trapezoidal neutrosophic number environment. Complex Intell. Syst. 5 (2019) 379-389.

[33] X. Sang and X. Liu, An analytic approach to obtain the least square deviation OWA operator weights. Fuzzy Set. Syst. 240 (2014) 103-116.

[34] A. Shaygan and O.M. Testik, A fuzzy AHP-based methodology for project prioritization and selection. Soft Comput. 23 (2019) $1309-1319$.

[35] F. Smarandache, Neutrosophic set, a generalisation of the intuitionistic fuzzy sets. J. New Theory 29 (2019) 01-35.

[36] F. Smarandache, Neutrosophy and neutrosophic logic. In: First International Conference on Neutrosophy, Neutrosophic Logic, Neutrosophic Set, Neutrosophic Probability and Statistics. University of New Mexico, Gallup, USA (2002) 1-147.

[37] R. Sumathi and C.A.C. Sweety, New approach on differential equation via trapezoidal neutrosophic number. Complex Intell. Syst. 5 (2019) 417-424.

[38] M. Touqeer, J. Salma and I. Rida, A grey relational projection method for multi attribute decision making based on three trapezoidal fuzzy numbers. J. Intell. Fuzzy Syst. 38 (2020) 5957-5967.

[39] M. Touqeer, S. Kiran and I. Rida, Evaluation model for manufacturing plants with linguistic information in terms of three trapezoidal fuzzy numbers. J. Intell. Fuzzy Syst. 38 (2020) 5969-5978.

[40] M. Touqeer, H. Abid and A. Misbah, Multi-attribute decision making using grey relational projection method based on interval type-2 trapezoidal fuzzy numbers. J. Intell. Fuzzy Syst. 38 (2020) 5979-5986.

[41] H. Wang, P. Madiraju, R. Sunderraman and Y.Q. Zhang, Interval Neutrosophic Sets. Department of Computer Science, State University Atlanta, Georgia, USA (2004).

[42] H. Wang, F. Smarandache, R. Sunderraman and Y.Q. Zhang, Interval Neutrosophic Sets and Logic: Theory and Applications in Computing. Hexis, Arizona (2005).

[43] H. Wang, F. Smarandache, R. Sunderraman and Y. Zhang, Single valued neutrosophic sets. Rev. Air Force Acad. 1 (2010) $10-14$.

[44] H. Wang, F. Smarandache, R. Sunderraman and Y.Q. Zhang, Single valued neutrosophic sets. Multispace Multistructure 4 (2010) 410-413.

[45] W. Wang, X. Liu and Y. Qin, Multi-attribute group decision making models under interval type-2 fuzzy environment. Knowl.-Based Syst. 30 (2012) 121-128.

[46] W. Weaver, Science and complexity. Am. Sci. 36 (1948) 536-544.

[47] G.W. Wei, H.J. Wang and R. Lin, Application of correlation coefficient to interval-valued intuitionistic fuzzy multiple attribute decision-making with incomplete weight information. Knowl. Inf. Syst. 26 (2011) 337-349.

[48] Z. Xu, An overview of methods of determining OWA weights. Int. J. Intell. Syst. 20 (2005) 843-865.

[49] Z. Xu, An integrated model-based interactive approach to FMAGDM with incomplete preference information. Fuzzy Optim. Decis. Making 9 (2010) 333-357.

[50] Z. Xu and R.R. Yager, Some geometric aggregation operators based on intuitionistic fuzzy sets. Int. J. Gen. Syst. 35 (2006) $417-433$.

[51] Y.Y. Yang, X. Wang and F. Liu, Trapezoidal interval type-2 fuzzy TOPSIS using alpha-cuts. Int. J. Fuzzy Syst. 22 (2020) 293-309.

[52] J.S. Yao and K. Wu, Ranking fuzzy numbers based on decomposition principle and signed distance. Fuzzy Set. Syst. 116 (2000) 275-288.

[53] J. Ye, Similarity measures between interval neutrosophic sets and their applications in multicriteria decision-making. J. Intell. Fuzzy Syst. 26 (2014) 165-172.

[54] J. Ye, Trapezoidal neutrosophic set and its application to multiple attribute decision making. Neural Comput. Appl. 26 (2015) 1157-1166.

[55] J. Ye, Some weighted aggregation operator of trapezoidal neutrosophic number and their multiple attribute decision making method. Informatica $\mathbf{2 8}$ (2015) 387-402.

[56] D. Yu, Intuitionistic trapezoidal tuzzy information aggregation methods and their applications to teaching quality evaluation. J. Inf. Comput. Sci. 10 (2013) 1861-1869. 
[57] L.A. Zadeh, Fuzzy sets. Inf. Control 8 (1965) 338-353.

[58] L.A. Zadeh, The concept of a linguistic variable and its application to approximate reasoning. Inf. Sci. 8 (1965) 199-249.

\section{Subscribe to Open (S20)} A fair and sustainable open access model

This journal is currently published in open access under a Subscribe-to-Open model (S2O). S2O is a transformative model that aims to move subscription journals to open access. Open access is the free, immediate, online availability of research articles combined with the rights to use these articles fully in the digital environment. We are thankful to our subscribers and sponsors for making it possible to publish this journal in open access, free of charge for authors.

\section{Please help to maintain this journal in open access!}

Check that your library subscribes to the journal, or make a personal donation to the $\mathrm{S} 2 \mathrm{O}$ programme, by contacting subscribers@edpsciences.org

More information, including a list of sponsors and a financial transparency report, available at: https://www. edpsciences.org/en/maths-s2o-programme 